\title{
GEOMETRY ASSOCIATED WITH SEMISIMPLE FLAT HOMOGENEOUS SPACES $\left({ }^{1}\right)$
}

\author{
BY \\ TAKUSHIRO OCHIAI
}

\begin{abstract}
Our object is Cartan connections with semisimple flat homogeneous spaces as standard spaces. We study these from the viewpoint of $G$-structures of second order. This allows us especially to treat classical projective and conformal connections in the unifying manner. We also consider its application to the problem of certain geometric transformations.
\end{abstract}

Introduction. Let $M$ be a manifold of dimension $n$. We denote by $\mathscr{F}^{1}(M)$ the frame bundle of $M$, and by $A\left(\mathscr{F}^{1}(M)\right)$ the set of all linear connections without torsion on $\mathscr{F}^{1}(M)$. The projective geometry of linear connections is the study of invariants of projectively equivalent classes in $A\left(\mathscr{F}^{1}(M)\right)$. It is the classical theory by $\mathrm{E}$ : Cartan that for each projectively equivalent class there corresponds uniquely a projective Cartan connection [4].

Examining the algebraic structure of projective spaces, N. Tanaka introduced the notion of a $l$-system $\mathfrak{l}$ and the homogeneous space $M_{\mathfrak{l}}=L(\mathfrak{l}) / L_{0}(\mathfrak{l})$ [29]. Generalizing the notion of projective equivalence, he gave the notion of $l$-equivalence in the set $A(P)$ of all $G_{0}(\mathfrak{l})$-connections without torsion on a given $G_{0}(\mathfrak{l})$ structure $P$ over $M$, where $G_{0}(\mathfrak{l})$ is the linear isotropy group of $M=L(\mathfrak{l}) / L_{0}(\mathfrak{l})$. Then he showed that for each $\mathfrak{l}$-equivalence class there corresponds uniquely a Cartan connection of type $M_{\mathfrak{l}}[29]$.

On the other hand S. Kobayashi and T. Nagano found that the projective equivalence is also defined by using the bundle $\mathscr{F}^{2}(M)$ of 2 nd order frames of $M$ [13]. And they reconstructed the above theory of E. Cartan [13].

Our first purpose in this paper is to rebuild the theory of N. Tanaka from the viewpoint of 2 nd order structures, thus generalizing the results in [13]. The Spencer cohomology group of a graded Lie algebra plays an important role in the constructions.

Next, we deal with the geometric tensors associated with our spaces. As one of the examples, a generalization of Weyl's projective tensors is given. It is proved that its vanishing is equivalent to the integrability of certain geometric structures.

Received by the editors November 3, 1969.

AMS 1969 subject classifications. Primary 5352, 5350, 5373.

Key words and phrases. Graded Lie algebra, Spencer cohomology, semisimple Lie algebra, harmonic class, semisimple flat homogeneous space, bundle of higher order contact, linear connection, Cartan connection, normal Cartan connection, Weyl tensor.

(1) Submitted to the Graduate School of the University of Notre Dame in partial fulfillment of the requirement for the degree of Doctor of Philosophy, June 1969.

Copyright (C) 1970, American Mathematical Society 
Our geometry depends heavily upon the theory of semisimple graded Lie algebras. $\$ 1$ through 7 are devoted to a study of graded Lie algebras and their Spencer cohomology groups in detail for our construction and theorem.

As a final purpose, we give some applications to the problem of geometric transformations. We generalize the well-known relationship between the group of isometries and that of projective transformations of a riemannian manifold with a parallel Ricci-tensor (cf. [20] and [21]).

The author wishes to express hearty thanks to his thesis advisor Professor T. Nagano for his encouragement and valuable suggestions. He is also very thankful to Professor Y. Matsushima, H. Ozeki and N. Tanaka.

Notations. Throughout the paper the following standard conventions have been adopted. $\boldsymbol{Z}$ denotes the ring of integers. $\boldsymbol{R}$ (resp. $\boldsymbol{C}$ ) denotes the field of real (resp. complex) numbers and $\boldsymbol{F}$ denotes $\boldsymbol{R}$ or $\boldsymbol{C}$. For a finite dimensional vector space $V$ over $\boldsymbol{F}$, we denote by $V^{*}$ the dual space of $V$. Also we denote with pointed brackets $(x \cdot f)$ the value of the canonical bilinear mapping $b: V \times V^{*} \rightarrow F$ taken at $\alpha \in V$ and $f \in V^{*}$. We denote by $\Lambda^{p} V$ (resp. $\Lambda V$ ) the $p$ th exterior product of $V$ (resp. the sum $\sum \Lambda^{p} V$ ). For a real vector space $V, V^{C}$ is the complexification of $V$, and for a complex vector space $V, V_{R}$ is the restriction of the field to $\boldsymbol{R}$.

For a point $x$ of a manifold $M, T(M)_{x}$ is the tangent space of $M$ at $x$. For a differentiable mapping $f, f_{*}$ and $f^{*}$ are the differential and the codifferential of $f$ respectively. For a Lie group $G$, its Lie algebra is written by the corresponding German letter $\mathfrak{g}$. The adjoint representation of $G$ (resp. $\mathfrak{g}$ ) into $g$ is denoted by Ad (resp. ad). For a vector space $V$ over $F, G L(V)$ denotes the general linear group, and $\operatorname{gl}(V)$ denotes its Lie algebra. For a $G$-principal bundle $P \rightarrow M, R_{a}$ denotes the right action of an element $a$ of $G$ on $P$. For an element $A$ of $\mathfrak{g}, A^{*}$ denotes the fundamental vector field on $P$ corresponding to $A$.

1. Outline of the results. We consider the $l$-system due to $N$. Tanaka from a different aspect, that is, from the viewpoint of graded Lie algebras (cf. [26]).

(a) A homogeneous space $L / L_{0}$ is called flat of order $r$ if the Lie algebra $\mathfrak{l}$ of $L$ has a graded Lie algebra structure $\mathfrak{I}=\sum_{p \in Z} \mathfrak{g}_{p}\left(\mathfrak{g}_{p}=0\right.$ if and only if $p \leqq-2$ or $\left.p \geqq r\right)$ such that $\mathfrak{I}_{0}=\sum_{p \geqq 0} \mathfrak{g}_{p}$ is the Lie subalgebra corresponding to $L_{0} . L / L_{0}$ is called semisimple flat if $L$ is furthermore semisimple. For a semisimple flat homogeneous space $L / L_{0}$, we shall prove:

(a-1) the order is two, i.e., $\mathfrak{l}=\mathfrak{g}_{-1} \oplus \mathfrak{g}_{0} \oplus \mathfrak{g}_{1}$;

(a-2) $L_{0}$ is a semidirect product $L_{0}=G_{0}^{\prime} \cdot G_{1}$ of $G_{0}^{\prime}$ and a connected Lie subgroup $G_{1}$ such that the Lie algebra of $G_{0}^{\prime}$ and $G_{1}$ are $g_{0}$ and $g_{1}$ respectively;

(a-3) $G_{1}$ is identical to the kernel of the linear isotropy representation, i.e., $G_{0}^{\prime}$ is isomorphic to the linear isotropy group $G_{0}$. By this we identify $G_{0}^{\prime}$ and $G_{0}$.

Homogeneous spaces $M_{\mathfrak{l}}$ defined by $\mathrm{N}$. Tanaka are examples of semisimple flat homogeneous spaces. For those $M_{\mathfrak{l}}$, the above results were proved in [29].

(b) $\mathfrak{g}_{-1}$ is an abelian Lie subalgebra of $\mathfrak{l}$. We consider the Lie algebra cohomology 
groups $H\left(\mathfrak{g}_{-1}\right.$, ad, $\left.\mathfrak{l}\right)=\sum_{q \geqq 0} H^{q}\left(\mathfrak{g}_{-1}\right.$, ad, $\left.\mathfrak{l}\right)$ of $\mathfrak{g}_{-1}$ with respect to its adjoint representation in $\mathfrak{l}$. The graded Lie algebra structure $\mathfrak{l}=\mathfrak{g}_{-1} \oplus \mathfrak{g}_{0} \oplus \mathfrak{g}_{1}$ induces another decomposition

$$
H^{q}\left(\mathfrak{g}_{-1}, \text { ad, } \mathfrak{l}\right)=\sum_{p \geqq 0} H^{p, q}\left(\mathfrak{g}_{-1}, \text { ad, } \mathfrak{l}\right) \quad \text { for } q \geqq 0 .
$$

The cohomology groups $H\left(\mathrm{~g}_{-1}^{\mathcal{C}}\right.$, ad $\left.{ }^{C}, \mathfrak{l}^{C}\right)=\sum H^{q}\left(\mathrm{~g}_{-1}^{\mathcal{C}}\right.$, ad $\left.{ }^{C}, \mathfrak{l}^{C}\right)$ has been studied by B. Kostant [16] and Y. Matsushima-S. Murakami [17], using a certain harmonic theory with respect to the canonically defined inner-product. Using their results, we shall prove:

(b-1) $H^{2,1}\left(\mathfrak{g}_{-1}\right.$, ad, l) vanishes for almost all $L / L_{0}$;

(b-2) $H^{2,2}\left(g_{-1}\right.$, ad, $\left.\mathfrak{l}\right)$ vanishes for almost all $L / L_{0}$; and we shall give

(b-3) the necessary and sufficient condition for $H^{1,1}\left(g_{-1}\right.$, ad, $\left.\mathfrak{l}\right)$ to vanish.

The equivalent statements of (b-1) and (b-3) are shown also in [29].

(c) The results of (a) permit us to consider the principal bundle $L \rightarrow L / L_{0}$ as a subbundle of the bundle of frames of 2 nd order contact of $L / L_{0}$. For a manifold $M$, we denote by $\mathscr{F}^{r}(M)$ the bundle of frames of $r$ th order contact [12]. Its structure group is denoted by $G^{r}(n)$ if $\operatorname{dim} M=n . \mathscr{F}^{1}(M)$ is usually called the frame bundle of $M$ and $G^{1}(n)$ is isomorphic to $G L(n, R) . G L(n, R)$ can be considered as a subgroup of $G^{r}(n)$. We shall prove:

(c-1) $L_{0}=G_{0} \cdot G_{1}$ is a subgroup of $G^{2}(n)$;

(c-2) $L_{0} \cap G L(n, R)=G_{0}$;

(c-3) $L \rightarrow L / L_{0}$ is a $L_{0}$-subbundle of $\mathscr{F}^{2}\left(L / L_{0}\right) \rightarrow L / L_{0}$.

(d) Let $P$ be a $G_{0}$-structure on $M$ i.e., a $G_{0}$-subbundle of $\mathscr{F}^{1}(M)$. From a theorem of S. Kobayashi [12], each $G_{0}$-connection on $P$ without torsion gives a $G_{0}$-subbundle of $\mathscr{F}^{2}(M) \rightarrow M$. Since $G^{2}(n) \supset L_{0} \supset G_{0}$, we have a $L_{0}$-subbundle of $\mathscr{F}^{2}(M)$ $\rightarrow M$ extending the structure group. Thus each $G_{0}$-connection $\Gamma$ on $P$ without torsion (if it exists) naturally gives a $L_{0}$-subbundle $Q(\Gamma)$ of $\mathscr{F}^{2}(M) \rightarrow M$. The above consideration gives the $L_{0}$-equivalence relation in the set $A(P)$ of all $G_{0}$-connections on $P$ without torsions as follows: two elements $\Gamma$ and $\Gamma^{\prime}$ of $A(P)$ are equivalent if and only if $Q(\Gamma)=Q\left(\Gamma^{\prime}\right)$. It is easy to see that the above $L_{0}$-equivalence is equivalent to the $l$-equivalence due to N. Tanaka [29]. Then we shall prove:

(d-1) A fixed equivalence class in $A(P)$ gives rise to a one-to-one correspondence between the equivalence class of $A(P)$ and $H^{1,1}\left(\mathfrak{g}_{-1}\right.$, ad, $\left.\mathfrak{l}\right)$.

(e) For each $L_{0}$-equivalence class $\mathfrak{a}$ in $A(P)$, we denote by $Q(\mathfrak{a})$ the $L_{0}$-subbundle of $\mathscr{F}^{2}(M) \rightarrow M$ defined by any element of $\mathfrak{a}$. $Q(\mathfrak{a})$ is called an $L_{0}$-structure of 2 nd order associated with $\mathfrak{a}$. From a theorem of $\mathrm{C}$. Ehresmann we easily see that there exists at least one Cartan connection on $Q(\mathfrak{a})$ of type $L / L_{0}$, i.e., an infinitesimal connection (satisfying one additional condition) on the $L$-principal bundle associated with the $L_{0}$-principal bundle $Q(\mathfrak{a})$. We can single out the most natural one among others by the following results:

(e-1) For each Cartan connection $\omega$ on $Q(a)$ its curvature defines a cocycle 
$c(\omega)_{x}$ in $H^{1,2}\left(\mathfrak{g}_{-1}\right.$, ad, $\left.\mathfrak{l}\right)(x \in Q(\mathfrak{a}))$ and for each point $x \in Q(\mathfrak{a})$ its cohomology class is independent of the choice of a particular $\omega$;

(e-2) If $H^{2,1}\left(\mathfrak{g}_{-1}\right.$, ad, $\left.\mathfrak{l}\right)=0$, there exists one and only one Cartan connection $\omega$ such that $c(\omega)_{x}$ is harmonic for each point $x \in Q(\mathfrak{a})$ (see (b)). We call the Cartan connection in (e-2) the normal Cartan connection of an $L_{0}$-equivalence class $a$. The above construction of normal Cartan connections seems to give a better interpretation of $\mathrm{N}$. Tanaka's normal Cartan connection of type $M_{\mathrm{r}}$.

(f) Using the normal Cartan connection, we shall define the Weyl tensor $W(\mathfrak{a})$ of an $L_{0}$-equivalence class $\mathfrak{a}$. $W(\mathfrak{a})$ is a tensor of type (1.3) on $M$. We shall prove:

(f-1) $Q(\mathfrak{a}) \rightarrow M$ is locally equivalent to $L \rightarrow L / L_{0}$ if and only if $W(\mathfrak{a})$ vanishes, provided $H^{2,2}\left(\mathfrak{g}_{-1}\right.$, ad, $\left.\mathfrak{l}\right)=0$.

If $H^{1,1}\left(\mathfrak{g}_{-1}\right.$, ad, $\left.\mathfrak{l}\right)=0$, then by (d-1) we have the well-defined tensor $W(P)$ for each $G_{0}$-structure provided $A(P)$ is not empty. We shall prove:

(f-2) If $H^{1,1}\left(\mathfrak{g}_{-1}\right.$, ad, $\left.\mathfrak{l}\right)=0, G_{0}$-structure $P$ is locally flat if and only if $W(P)=0$.

(g) Let $\Gamma$ be an element of an $L_{0}$-equivalence class $\mathfrak{a}$. We denote by Aut $(P, \mathfrak{a})$ (resp. Aut $(P, \Gamma)$ ) the group of all $G_{0}$-automorphisms preserving a (resp. $\Gamma$ ). It is plausible to conjecture: if $\Gamma$ is Ricci-parallel and $\operatorname{Aut}(P, \mathfrak{a}) \neq \operatorname{Aut}(P, \Gamma), M$ is diffeomorphic to $L / L_{0}$ and Aut $(P, \mathfrak{a})=L$ (up to covering). We shall prove the conjecture under some restrictive additional conditions:

(g-1) If Aut $(P, \mathfrak{a})$ is transitive and irreducible, the above conjecture is true.

(h) Let us point out some connections with classical results. Examples of semisimple flat homogeneous spaces are given by:

(1) a projective space and the group of its projective transformations, and

(2) a Moebius space and the group of its conformal transformations.

When $L / L_{0}$ is a projective space, $G_{0}$ is $G L(n, R)$ and $L_{0}$-equivalence is the projective equivalence. For a projective equivalence class $\mathfrak{a}$, our Weyl tensor $W(\mathfrak{a})$ coincides with the classical Weyl's projective tensor [30]. When $L / L_{0}$ is a Moebius space, $G_{0}$ is $\mathrm{CO}(n)$. In this case we have $H^{1,1}\left(\mathfrak{g}_{-1}\right.$, ad, $\left.\mathfrak{l}\right)=0$. Then the Weyl tensor $W(P)$ for any $\mathrm{CO}(n)$-structure coincides with the classical Weyl's conformal tensor [30]. It is easily seen that in both cases our conjecture is true from [20] and [21].

2. Graded Lie algebras and Spencer cohomologies. A finite dimensional Lie algebra $\mathfrak{g}$ over $\boldsymbol{F}(\boldsymbol{H}=\boldsymbol{C}$ or $\boldsymbol{R})$, is called a graded Lie algebra over $\boldsymbol{F}$ if $\mathfrak{g}$ is a direct sum of subspaces $\mathfrak{g}_{p}(p \in Z)$ such that

(2-1). $\mathfrak{g}_{p}=0$ for $p \leqq-2$,

(2-2). $\left[\mathfrak{g}_{p}, \mathfrak{g}_{q}\right] \subset \mathfrak{g}_{p+q}$ for all $p$ and $q$,

(2-3). for an $x \in \mathfrak{g}_{p}(p \geqq 0, x \neq 0),\left[\mathfrak{g}_{-1}, x\right] \neq 0$.

Two graded Lie algebras (over $\boldsymbol{F}$ ) $\mathfrak{g}=\sum \mathfrak{g}_{p}$ and $\mathfrak{g}^{\prime}=\sum \mathfrak{g}_{p}^{\prime}$ are called isomorphic if there exists a Lie algebra isomorphism $\phi: \mathfrak{g} \rightarrow \mathfrak{g}^{\prime}$ such that $\phi\left(\mathfrak{g}_{p}\right)=\mathfrak{g}_{p}^{\prime}$ for all $p$. By a graded Lie subalgebra $\mathfrak{h}=\sum \mathfrak{h}_{p}$ of the graded Lie algebra $\mathfrak{g}=\sum \mathfrak{g}_{p}$, we mean a subalgebra $\mathfrak{h}$ of $\mathfrak{g}$ such that $\mathfrak{h}_{p} \subset \mathfrak{g}_{p}$. If $\mathfrak{g}_{p} \neq 0$, then from (2-3) we have $\mathfrak{g}_{q} \neq 0$ for $-1 \leqq q \leqq p$. Thus there exists uniquely an integer $r$ such that $\mathfrak{g}_{p} \neq 0$ for $-1 \leqq p \leqq r-1$ 
and $\mathfrak{g}_{p}=0$ for $p \geqq r$. The integer $r$ is called the order of the graded Lie algebra $\mathfrak{g}=\sum \mathfrak{g}_{p}$. For two graded Lie algebras $\mathfrak{g}=\sum \mathfrak{g}_{p}$ and $\mathfrak{g}^{\prime}=\sum \mathfrak{g}_{p}^{\prime}$ we define their direct sum $\tilde{\mathfrak{g}}=\sum \tilde{\mathfrak{g}}_{p}$ in the following natural manner; $\tilde{\mathfrak{g}}=\mathfrak{g} \oplus \mathfrak{g}^{\prime}$ (direct sum) and $\tilde{\mathfrak{g}}_{p}=$ $\mathfrak{g}_{p} \oplus \mathfrak{g}_{p}^{\prime}$ (direct sum). For a graded Lie algebra $\mathfrak{g}=\sum \mathfrak{g}_{p}$ over $\boldsymbol{R}$ (resp. $\boldsymbol{C}$ ) we can naturally define its complexification $\mathrm{g}^{C}$ (resp. the restriction of the field $\mathrm{g}_{R}$ ).

LEMMA 2-1. For a graded Lie algebra $\mathrm{g}=\sum \mathrm{g}_{p}$, we have

(2-4). $\mathfrak{g}_{-1}$ is an abelian Lie subalgebra of $\mathfrak{g}$,

(2-5). $\mathrm{g}_{0}$ is a Lie subalgebra of $\mathrm{g}$,

(2-6). $\sum_{p \geq 0} \mathfrak{g}_{p}$ is a Lie subalgebra of $\mathfrak{g}$,

(2-7). $\sum_{p \geqq k} \mathfrak{g}_{p}$ is an ideal of $\sum_{p \geqq 0} \mathfrak{g}_{p}$,

(2-8). the adjoint representation $\operatorname{ad}_{\mathfrak{g}} \mathfrak{g}_{0}$ of $\mathfrak{g}_{0}$ into $\mathfrak{g}$ leaves each $\mathfrak{g}_{p}$ invariant.

Proof. These are trivial from the definition.

By (2-8) we have a representation $\operatorname{ad}_{\mathfrak{g}} \mathfrak{g}_{0} \mid \mathfrak{g}_{p}$ of $\mathfrak{g}_{0}$ into $\mathfrak{g}_{p}$. The representation $\operatorname{ad}_{\mathfrak{g}} \mathfrak{g}_{0} \mid \mathfrak{g}_{-1}$ is especially called the linear isotropy representation of $\mathfrak{g}=\sum \mathfrak{g}_{p}$. By (2-3) this representation is faithful. The graded Lie algebra is called irreducible if its linear isotropy representation is irreducible.

For a graded Lie algebra (over $\boldsymbol{F}) \mathfrak{g}=\sum \mathfrak{g}_{p}$, the Lie algebra cohomology $H(\mathfrak{g})\left({ }^{2}\right)$ $=H\left(\mathfrak{g}_{-1}, \operatorname{ad}_{\mathfrak{g}} \mid \mathfrak{g}_{-1}, \mathfrak{g}\right)$ of the abelian Lie algebra $\mathfrak{g}_{-1}$ with respect to its adjoint representation into $\mathfrak{g}$ is called the Spencer cohomology of $\mathfrak{g}=\sum \mathfrak{g}_{p}$. With the graded structure in mind, let us recall the definition. Let $C^{p, q}(g)$ be the vector space of all $\mathfrak{g}_{p-1}$-valued $q$-forms on $\mathfrak{g}_{-1}$ (i.e., $C^{p, q}(\mathfrak{g})=\mathfrak{g}_{p-1} \otimes \Lambda^{q}\left(\mathfrak{g}_{-1}\right)^{*}$ ) and put $C(\mathfrak{g})=$ $\sum C^{p, q}(\mathfrak{g})$. Now we define coboundary operator $\partial: C(\mathfrak{g}) \rightarrow C(\mathfrak{g})$ to be $\partial C^{p, q}(\mathfrak{g})$ $\subset C^{p-1, q+1}(g)$ and

(2-9). $(\partial c)\left(x_{1}, \ldots, x_{q+1}\right)=\sum_{i=1}^{q+1}(-1)^{i+1}\left[x_{i}, c\left(x_{1}, \ldots, \hat{x}_{i}, \ldots, x_{q+1}\right)\right]$ for all $c \in$ $C^{p, q}(\mathfrak{g})$ and $x_{1}, \ldots, x_{q+1} \in \mathfrak{g}_{-1}$. Then we have $\partial^{2}=0$. Thus we have the cohomology $H(g)$ by

$(2-10) . H(g)=\partial^{-1}(0) / \partial(g)$.

\section{Putting}

$(2-11) . H^{p, q}(\mathfrak{g})=\partial^{-1}(0) \cap C^{p, q}(\mathfrak{g}) / \partial\left(C^{p+1, q-1}(\mathfrak{g})\right), H(\mathfrak{g})$ is naturally isomorphic to $\sum H^{p, q}(\mathfrak{g})$.

LEMMA 2-2. We have

(2-12). $H^{0,0}(\mathrm{~g}) \cong \mathfrak{g}_{-1}$,

(2-13). $H^{p, 0}(\mathfrak{g})=0$ if $p \neq 0$,

(2-14). $H^{0,1}(\mathfrak{g}) \cong \mathfrak{g l}\left(\mathfrak{g}_{-1}\right) / \mathfrak{g}_{0}$.

Proof. These are easy to see. (2-13) comes from (2-3).

The following are standard facts on Lie algebra cohomologies [25].

${ }^{(2)}$ We should write $H\left(\Sigma \mathfrak{g}_{p}\right)$. But we take this notation if there is no danger of confusion. 
LEMMA 2-3. Let $\tilde{\mathrm{g}}=\sum \tilde{\mathrm{g}}_{p}$ be the direct sum of two graded Lie algebras $\mathfrak{g}=\sum \mathfrak{g}_{p}$ and $\mathfrak{g}^{\prime}=\sum \mathfrak{g}_{p}^{\prime}$. Then we have

$$
H^{p, q}(\tilde{\mathfrak{g}})=\left(\sum_{s+t=q} H^{p, s}(\mathfrak{g}) \otimes \Lambda^{t}\left(\mathfrak{g}_{-1}^{\prime}\right)^{*}\right) \oplus\left(\sum_{s+t=q} \Lambda^{s}\left(\mathfrak{g}_{-1}\right)^{*} \otimes H^{p, t}\left(\mathfrak{g}^{\prime}\right)\right) .
$$

LEMMA 2-4. Let $\mathfrak{g}^{C}$ be the complexification of a real graded Lie algebra $\mathfrak{g}=\sum \mathfrak{g}_{p}$. Then we have $H^{p, q}\left(\mathfrak{g}^{C}\right) \cong H^{p, q}(\mathfrak{g}) \otimes C$ for all $p, q$.

The Lie subalgebra $g_{0}$ has a natural representation $\rho$ on each $C^{p, q}(g)$. Then it is easy to see

LEMMA 2-5. We have $\partial(\rho(a) c)=\rho(a)(\partial c)$ for all $c \in C(\mathfrak{g})$ and $a \in \mathfrak{g}_{0}$.

By the above lemma, there is a natural representation $\rho$ of $g_{0}$ into

$$
H(\mathrm{~g})=\sum H^{p, q}(\mathrm{~g}) .
$$

3. Semisimple graded Lie algebras. A graded Lie algebra $\mathfrak{g}=\sum \mathfrak{g}_{p}$ over $\boldsymbol{F}$ is called semisimple (resp. simple) if $\mathfrak{g}$ is semisimple (resp. simple) over $\boldsymbol{F}$. Unless otherwise stated, we assume, in this section, all graded Lie algebras considered are semisimple (or simple) over $\boldsymbol{F}$.

Proposition 3-1. The order of $\mathrm{g}=\sum \mathrm{g}_{p}$ is 2 .

Proof. Take $z \in \mathfrak{g}_{2}$. Then ad $x$ ad $z: \mathfrak{g} \rightarrow \mathfrak{g}$ is nilpotent for all $x \in \mathfrak{g}$. Denoting by $B$ the Killing form of $\mathfrak{g}$, we have $B(x, \mathfrak{g})=0$. Since $B$ is nondegenerate, we have $x=0$.

Lemma 3-1. The linear endomorphism $\alpha$ of $\mathfrak{g}=\mathfrak{g}_{-1} \oplus \mathfrak{g}_{0} \oplus \mathfrak{g}_{1}$ defined by

$$
\alpha(x+y+z)=-x+y-z \text { for } x \in \mathfrak{g}_{-1}, y \in \mathfrak{g}_{0}, z \in \mathfrak{g}_{1},
$$

is an involutive automorphism of $\mathrm{g}$.

Proof. The proof is straightforward.

Lemma 3-2. Denote by $B$ the Killing form of $\mathrm{g}$. Then

(3-1). $\mathfrak{g}_{-1} \oplus \mathfrak{g}_{1}$ and $\mathfrak{g}_{0}$ are perpendicular to each other with respect to $B$, so that both $B \mid \mathfrak{g}_{-1} \oplus \mathfrak{g}_{1}$ and $B \mid \mathfrak{g}_{0}$ are nondegenerate;

(3-2). $B \mid \mathfrak{g}_{-1}=0$ and $B \mid \mathfrak{g}_{1}=0$;

(3-3). $\mathfrak{g}_{-1}$ is the dual vector space of $\mathfrak{g}_{1}$ under the pairing $(x, z) \rightarrow B(x, z)$ $\left(x \in \mathfrak{g}_{-1}, z \in \mathfrak{g}_{1}\right)$.

Proof. (i) Let $\alpha$ be the involutive automorphism of $g$ defined in Lemma (3-1). Since $B$ is invariant by $\alpha$, we have

$$
B(x+z, y)=B(\alpha(x+z), \alpha(y))=B(-x-z, y) \quad \text { for } x \in \mathfrak{g}_{-1}, y \in \mathfrak{g}_{0} \text { and } z \in \mathfrak{g}_{1} .
$$

Hence $\mathfrak{g}_{-1} \oplus \mathfrak{g}_{1}$ and $g_{0}$ are perpendicular with respect to $B$. Thus, since $B$ is nondegenerate, so are its restrictions to $\mathfrak{g}_{-1} \oplus \mathfrak{g}_{1}$ and $\mathfrak{g}_{0}$. 
(ii) Let $x, x^{\prime} \in \mathfrak{g}_{-1}$. Then ad $x$ ad $x^{\prime}: \mathfrak{g} \rightarrow \mathfrak{g}$ is nilpotent. Hence $B\left(x, x^{\prime}\right)=0$ proving $B \mid \mathfrak{g}_{-1}=0$. Similarly $B \mid \mathfrak{g}_{1}=0$.

(iii) Let $x \in \mathfrak{g}_{-1}$, and assume $B\left(x, \mathfrak{g}_{1}\right)=0$. By (3-1) and (3-2), this implies $B(x, \mathfrak{g})$ $=0$. Hence $x=0$. Similarly, if $z \in \mathfrak{g}_{1}$, and $B\left(\mathfrak{g}_{-1}, z\right)=0$, then $z=0$.

LemMA (3-3). The representation $\operatorname{ad}_{\mathfrak{g}} \mathfrak{g}_{0} \mid \mathfrak{g}_{-1}$, and $\operatorname{ad}_{\mathfrak{g}} \mathfrak{g}_{0} \mid \mathfrak{g}_{1}$ of $\mathfrak{g}_{0}$ are dual to each other with respect to the Killing form $B$.

Proof. This follows from the invariance of $B$ under ad $y\left(y \in g_{0}\right)$, i.e.

$$
B(\operatorname{ad}(y) x, z)=-B(x, \operatorname{ad}(y) z) \text { for } x \in \mathfrak{g}_{-1}, z \in \mathfrak{g}_{1} .
$$

LEMma (3-4). There exists uniquely an element $e$ in $\mathrm{g}_{0}$ such that

$$
\begin{array}{ll}
{[e, x]=-x} & \text { for } x \in \mathfrak{g}_{-1}, \\
{[e, y]=0} & \text { for } y \in \mathfrak{g}_{0}, \\
{[e, z]=z} & \text { for } z \in \mathfrak{g}_{1} .
\end{array}
$$

Proof [14]. Let $E$ be the linear endomorphism defined by $E(x)=-x$ for $x \in \mathfrak{g}_{-1}, E(y)=0$ for $y \in \mathfrak{g}_{0}$ and $E(z)=z$ for $z \in \mathfrak{g}_{1}$. Then it is easy to see that $E$ is a derivation of $\mathfrak{g}$. Since every derivation of a semisimple Lie algebra is inner, there is a unique element $e$ in $g$ such that $E=$ ad $e$ (the uniqueness follows from the fact that $g$ has no center). Let $\alpha$ be the involutive automorphism defined in Lemma (3-1). We can easily see that ad $(\alpha(e))$ coincides with $E$. Hence $\alpha(e)=e$ proving $e \in g_{0}$.

COROllary (3-1). An abelian Lie subalgebra $\mathfrak{a}$ of $\mathfrak{g}$ containing e (of Lemma (3-4)) is contained in $\mathfrak{g}_{0}$. In particular there exists a Cartan subalgebra $\mathfrak{h}$ of $\mathfrak{g}$ contained in $g_{0}$.

Proof. Take an element $a=x+y+z\left(a \in \mathfrak{a}, x \in \mathfrak{g}_{-1}, y \in \mathfrak{g}_{0}, z \in \mathfrak{g}_{1}\right)$. Then $0=$ $[e, a]=[e, x+y+z]=-x+z$. Hence $x=0, z=0$, i.e., $\mathfrak{a} \subset \mathfrak{g}_{0}$. Since ad $e$ is $\boldsymbol{F}$ diagonalizable, there exists a Cartan subalgebra $\mathfrak{h}$ containing $e . \mathfrak{h}$ being abelian, we get $\mathfrak{h} \subset \mathfrak{g}_{0}$.

Proposition (3-2) [29]. (i) Let $\mathrm{g}=\mathfrak{\Im}_{1} \oplus \cdots \oplus \mathfrak{\Im}_{r}$ be the decomposition of $\mathfrak{g}$ into its simple factors. Then

$$
\Im_{j}=\left(\mathfrak{g}_{-1} \cap \Im_{j}\right) \oplus\left(g_{0} \cap \Im_{j}\right) \oplus\left(\mathfrak{g}_{1} \oplus \Im_{j}\right)
$$

and $\mathfrak{\Im}_{j}=\sum\left(\mathfrak{g}_{p} \cap \mathfrak{\Im}_{j}\right)$ is a simple graded Lie algebra.

(ii) $\mathfrak{g}=\sum \mathfrak{g}_{p}$ is isomorphic to the direct sum of $\mathfrak{\Im}_{j}=\sum\left(\mathfrak{g}_{p} \cap \mathfrak{\Im}_{j}\right)(j=1, \ldots, r)$.

Proof. Take an element $a=x+y+z\left(a \in \mathfrak{\Im}_{j}, x \in \mathfrak{g}_{-1}, y \in \mathfrak{g}_{0}, z \in \mathfrak{g}_{1}\right)$. Then $\mathfrak{\Im}_{j} \ni$ $[e, a]=-x+z$ and $\mathfrak{\Im}_{j} \ni[e,[e, a]]=x+z$. Hence $\mathfrak{\Im}_{j}=\left(\mathfrak{g}_{-1} \cap \mathfrak{\jmath}_{j}\right) \oplus\left(\mathfrak{g}_{0} \cap \Im_{j}\right) \oplus$ $\left(g_{1} \cap \Im_{j}\right)$. The rest of our assertions are straightforward.

Proposition (3-3). A graded Lie algebra $\mathfrak{g}=\sum \mathfrak{g}_{p}$ is simple if and only if it is irreducible and the order is $>1$. 
Proof. See [14], [23] or [29].

Corollary (3-2). We have $B(x, z)=2\left(\right.$ the trace of ad $\left.[x, z] \mid \mathfrak{g}_{1}\right)$ for all $x \in \mathfrak{g}_{-1}$ and $z \in \mathfrak{g}_{1}$.

Proof [29]. 2(the trace of ad $\left.[x, z] \mid \mathfrak{g}_{1}\right)=B([x, z], e)=B(x,[z, e])=B(x, z)$.

COROLlaRY (3-3). We have $\left[\mathfrak{g}_{-1}, \mathfrak{g}_{1}\right]=\mathfrak{g}_{0}$ and $\mathfrak{g}_{0}$ is a reductive Lie algebra.

Proof. From Proposition (3-2), clearly we can assume that $\mathfrak{g}$ is simple. Then, by Proposition 3-3, $g_{0}$ has a faithful irreducible representation. Therefore $g_{0}$ must be reductive. It is easy to see that $\mathfrak{g}_{-1} \oplus\left[\mathfrak{g}_{-1}, \mathfrak{g}_{1}\right] \oplus \mathfrak{g}_{1}$ is an ideal of $\mathfrak{g}$. Therefore we have $\left[\mathfrak{g}_{-1}, \mathfrak{g}_{1}\right]=\mathfrak{g}_{0}$.

Proposition (3-4). $\mathfrak{g}=\mathfrak{g}_{-1} \oplus \mathfrak{g}_{0} \oplus \mathfrak{g}_{1}$ and $\mathfrak{g}^{\prime}=\mathfrak{g}_{-1}^{\prime} \oplus \mathfrak{g}_{0}^{\prime} \oplus \mathfrak{g}_{1}^{\prime}$ are isomorphic in the sense in $\S 2$ if and only if $\mathrm{g}_{0} \oplus \mathrm{g}_{1}$ and $\mathrm{g}_{0}^{\prime} \oplus \mathrm{g}_{1}^{\prime}$ are isomorphic as abstract Lie algebras.

Proof. Let $\phi: \mathfrak{g}_{0} \oplus \mathfrak{g}_{1} \rightarrow \mathfrak{g}_{0}^{\prime} \oplus \mathfrak{g}_{1}^{\prime}$ be an isomorphism. We denote by $\mathfrak{c}$ and $\mathfrak{c}^{\prime}$ the center of $g_{0}$ and $\mathfrak{g}_{0}^{\prime}$ respectively. Then clearly $\left[\mathfrak{g}_{0}, \mathfrak{g}_{0}\right] \oplus\left(\mathfrak{c} \oplus \mathfrak{g}_{1}\right)$ and $\left[\mathfrak{g}_{0}^{\prime}, \mathfrak{g}_{0}^{\prime}\right]$ $\oplus\left(\mathfrak{c}^{\prime}+\mathfrak{g}_{1}^{\prime}\right)$ are the Levi decomposition of $\mathfrak{g}_{0} \oplus \mathfrak{g}_{1}$ and $\mathfrak{g}_{0}^{\prime} \oplus \mathfrak{g}_{1}^{\prime}$ respectively. Since the Levi decomposition is unique up to conjugation, we can assume $\phi\left(\left[g_{0}, g_{0}\right]\right)$ $=\left[\mathfrak{g}_{0}^{\prime}, \mathfrak{g}_{0}^{\prime}\right]$ and $\phi\left(\mathfrak{c} \oplus \mathfrak{g}_{1}\right)=\mathfrak{c}^{\prime} \oplus \mathfrak{g}_{1}^{\prime}$, changing $\phi$ if necessary. Then we have

$$
\phi\left(\mathfrak{g}_{1}\right)=\phi\left(\left[\mathfrak{c} \oplus \mathfrak{g}_{1}, \mathfrak{c} \oplus \mathfrak{g}_{1}\right]\right)=\left[\mathfrak{c}^{\prime} \oplus \mathfrak{g}_{1}^{\prime}, \mathfrak{c}^{\prime} \oplus \mathfrak{g}_{1}^{\prime}\right]=\mathfrak{g}_{1}^{\prime} .
$$

We shall show that for a nonzero element $y$ of $c$ the $\mathfrak{c}^{\prime}$-component of $\phi(y)$ is not zero. In fact, otherwise, for any $z \in \mathfrak{g}_{1}$, we have $\phi([y, z])=[\phi(y), \phi(z)]=0$. Hence $[y, z]=0$ since $\phi\left(\mathfrak{g}_{1}\right)=\mathfrak{g}_{1}$. From this we get $y=0$. We define an isomorphism $\phi^{\prime}: \mathfrak{c} \rightarrow \mathfrak{c}^{\prime}$ by $\phi(y)=\phi^{\prime}(y) \bmod \mathfrak{g}_{1}^{\prime}$. Then we define a new mapping $\phi^{\prime \prime}:\left[\mathfrak{g}_{0}, \mathfrak{g}_{0}\right]$ $\oplus \mathfrak{c} \oplus \mathfrak{g}_{1} \rightarrow\left[\mathfrak{g}_{0}^{\prime}, \mathfrak{g}_{0}^{\prime}\right] \oplus \mathfrak{c}^{\prime} \oplus \mathfrak{g}_{1}^{\prime} \quad$ by $\quad \phi^{\prime \prime}(x+y+z)=\phi(x)+\phi^{\prime}(y)+\phi(z) \quad\left(x \in\left[\mathfrak{g}_{0}, \mathfrak{g}_{0}\right]\right.$, $y \in \mathfrak{c}$ and $\left.z \in \mathfrak{g}_{1}\right)$. It is easy to see that $\phi^{\prime \prime}$ is an isomorphism from $\mathfrak{g}_{0} \oplus \mathfrak{g}_{1}$ onto $\mathfrak{g}_{0}^{\prime} \oplus \mathfrak{g}_{1}^{\prime}$ such that $\phi^{\prime}\left(\mathfrak{g}_{0}\right)=\mathfrak{g}_{0}^{\prime}$ and $\phi^{\prime \prime}\left(\mathfrak{g}_{1}\right)=\mathfrak{g}_{1}^{\prime}$. Therefore we can assume that our isomorphism $\phi$ satisfies $\phi\left(\mathfrak{g}_{0}\right)=\mathfrak{g}_{0}^{\prime}$ and $\phi\left(\mathfrak{g}_{1}\right)=\mathfrak{g}_{1}^{\prime}$. Using the duality of $\mathfrak{g}_{-1}$ and $\mathfrak{g}_{1}$ (Lemma (3-2) and Lemma (3-3)), we can extend $\phi$ to a linear endomorphism from $\mathfrak{g}_{-1}$ onto $\mathfrak{g}_{-1}^{\prime}$. In fact, it suffices to show $\phi([x, z])=[\phi(x), \phi(z)]$ for $x \in \mathfrak{g}_{-1}, z \in \mathfrak{g}_{1}$. We denote by $B^{\prime}$ the Killing form of $\mathfrak{g}^{\prime}$. For $y \in g_{0}$, we have

$$
\begin{array}{rlr}
B^{\prime}(\phi([x, z]), \phi(y)) & =B([x, z], y) & \text { (easy to see) } \\
& =B(x,[z, y])=B^{\prime}(\phi(x), \phi([z, y])) & \text { (from the definition) } \\
& =B^{\prime}(\phi(x),[\phi(z), \phi(y)])=B^{\prime}([\phi(x), \phi(z)], \phi(y)) & \text { (Lemma 3-2). }
\end{array}
$$

Corollary (3-4). $\mathfrak{g}=\mathfrak{g}_{-1} \oplus \mathfrak{g}_{0} \oplus \mathfrak{g}_{1}$ and $\mathfrak{g}^{\prime}=\mathfrak{g}_{-1}^{\prime} \oplus \mathfrak{g}_{0}^{\prime} \oplus \mathfrak{g}_{1}^{\prime}$ are isomorphic if and only if $\mathrm{g}_{0}$ and $\mathrm{g}_{0}^{\prime}$ are isomorphic and their linear isotropy representations are equivalent.

Proof. This is a direct consequence of Proposition (3-4). 
LeMmA (3-5). Let $\mathfrak{g}=\mathfrak{g}_{-1} \oplus \mathfrak{g}_{0} \oplus \mathfrak{g}_{1}$ be real simple, If $\mathrm{g}^{C}$ is not simple, there exists a complex simple graded Lie algebra $\mathfrak{h}=\mathfrak{h}_{-1} \oplus \mathfrak{h}_{0} \oplus \mathfrak{h}_{1}$ such that $\mathfrak{g}=\mathfrak{h}_{\boldsymbol{R}}$. Further $\mathfrak{g}^{C} \cong \mathfrak{h} \oplus \mathfrak{h}$.

Proof. Since $\mathrm{g}^{C}$ is not simple, it is known that $\mathrm{g}$ is a complex simple Lie algebra. We shall show $g_{0}$ and $g_{1}$ are complex subalgebras. Let $\left[\mathfrak{g}_{0} \oplus \mathfrak{g}_{1}\right]$ be the minimal complex subalgebra of $\mathfrak{g}$ containing $\mathfrak{g}_{0} \oplus \mathfrak{g}_{1}$ and $\left\{\mathfrak{g}_{0} \oplus \mathfrak{g}_{1}\right\}$ be the maximal complex subalgebra contained in $\mathfrak{g}_{0} \oplus \mathfrak{g}_{1}$. Since $\mathfrak{g}=\mathfrak{g}_{-1} \oplus \mathfrak{g}_{0} \oplus \mathfrak{g}_{1}$ is irreducible, $\left[\mathfrak{g}_{0}+\mathfrak{g}_{1}\right]$ is either $\mathfrak{g}_{0} \oplus \mathfrak{g}_{1}$ or $\mathfrak{g}$. In the first case $\mathfrak{g}_{0} \oplus \mathfrak{g}_{1}$ is a complex subalgebra of $\mathfrak{g}$. Now suppose $\left[g_{0}+g_{1}\right]$ is $g$. Since $\left\{g_{0}+g_{1}\right\}$ is an ideal of $\left[g_{0}+g_{1}\right]$ (see the next lemma), [ $\left.g_{0} \oplus g_{1}\right]=0$, i.e., $g=\left(g_{0} \oplus g_{1}\right) \oplus \sqrt{ }-1\left(g_{0} \oplus g_{1}\right)$. This is absurd from the consideration of dimension (cf. (3-3)). Thus we have shown that $g_{0} \oplus g_{1}$ is a complex subalgebra of $g$. Since $\mathfrak{g}_{1}=\left\{y \in \mathfrak{g}_{0} \mid[\mathfrak{g}, y] \subset \mathfrak{g}_{0}\right\}, \mathfrak{g}_{1}$ is also a complex space. The rest of the proof is straightforward.

Lemma (3-6). $\left\{\mathfrak{g}_{0} \oplus \mathfrak{g}_{1}\right\}$ is an ideal of $\left[\mathfrak{g}_{0} \oplus \mathfrak{g}_{1}\right]$.

Proof. Let $x \in\left\{\mathfrak{g}_{0} \oplus \mathfrak{g}_{1}\right\}$ and $y \in \mathfrak{g}_{0} \oplus \mathfrak{g}_{1}$. Then for an arbitrary complex number $\lambda, \lambda[x, y]=[\lambda x, y] \in \mathfrak{g}_{0} \oplus \mathfrak{g}_{1}$. Therefore $[x, y] \in\left\{\mathfrak{g}_{0} \oplus \mathfrak{g}_{1}\right\}$. Now let $z \in\left[\mathfrak{g}_{0} \oplus \mathfrak{g}_{1}\right]$. Then $z=z_{1}+\sqrt{ }-1 z_{2}$, where $z_{1}, z_{2} \in g_{0} \oplus \mathfrak{g}_{1}$, and we have

$$
[x, z]=\left[x, z_{1}+\sqrt{ }-1 z_{2}\right]=\left[x, z_{1}\right]+\sqrt{ }-1\left[x, z_{2}\right] \in\left\{g_{0} \oplus g_{1}\right\} .
$$

Proposition (3-5). There exists an involutive automorphism $\sigma$ of $\mathrm{g}$ such that

$$
\sigma\left(\mathfrak{g}_{-1}\right)=\mathfrak{g}_{1}, \quad \sigma\left(\mathfrak{g}_{0}\right)=\mathfrak{g}_{0}, \quad \sigma\left(\mathfrak{g}_{1}\right)=\mathfrak{g}_{-1},
$$

and the bilinear form $B^{*}$ on $\mathrm{g}$ defined by $B^{*}(x, y)=-B(x, \sigma(y))(x, y \in \mathfrak{g}$ and $B$ is the Killing form of $\mathrm{g}$ ) is positive definite or positive definite hermitian symmetric, according as $\boldsymbol{F}=\boldsymbol{R}$ or $\boldsymbol{F}=\boldsymbol{C}$.

Proof. See [14] or [29].

Lemma (3-7). Let $\mathfrak{g}=\mathfrak{g}_{-1} \oplus \mathfrak{g}_{0} \oplus \mathfrak{g}_{1}$ be a simple graded Lie algebra over $\boldsymbol{F}$. Let $\mathfrak{h}$ be an irreducible subalgebra of $\mathrm{g}_{0}\left(\subset \mathrm{gl}\left(\mathrm{g}_{-1}\right)\right)$. If there exists a nonzero element $z$ in $\mathfrak{g}_{1}$ such that $\left[\mathfrak{g}_{-1}, z\right] \subset \mathfrak{h}$, then $\mathfrak{h}$ is necessary identical to $\mathfrak{g}_{0}$.

Proof. Let $\mathfrak{h}_{1}$ be $\left\{z^{\prime} \in \mathfrak{g}_{1} \mid\left[\mathfrak{g}_{-1}, z^{\prime}\right] \subset \mathfrak{h}\right\}$. Then clearly $\mathfrak{g}_{-1} \oplus \mathfrak{h} \oplus \mathfrak{h}_{1}$ is a graded Lie algebra. Since the linear isotropy representation is irreducible, $\mathfrak{g}_{-1} \oplus \mathfrak{h} \oplus \mathfrak{h}_{1}$ is simple by Proposition (3-3). Then by Lemma (3-2), the dimension of $\mathfrak{h}_{1}$ must be equal to that of $\mathfrak{g}_{-1}$. In particular we have $\mathfrak{h}_{1}=\mathfrak{g}_{1}$. Then by Corollary (3-3), we see $\mathfrak{h}_{0}=\mathfrak{g}_{0}$.

Proposition (3-6). Let $\mathfrak{g}=\mathfrak{g}_{-1} \oplus \mathfrak{g}_{0} \oplus \mathfrak{g}_{1}$ be a simple graded Lie algebra over $\boldsymbol{F}$. And let $\mathfrak{s}$ be a subalgebra of the Lie algebra $\mathrm{g}$ such that

(1) $\operatorname{dim} \mathfrak{g} /\left(\mathfrak{g}_{0} \oplus \mathfrak{g}_{1}\right)=\operatorname{dim} \mathfrak{g}_{-1}$, 
(2) the natural representation of $\mathfrak{s} \cap\left(\mathfrak{g}_{0} \oplus \mathfrak{g}_{1}\right)$ into $\mathfrak{s} /\left(\mathfrak{g}_{0} \oplus \mathfrak{g}_{1}\right)$ is irreducible,

(3) $\mathfrak{s} \cap \mathfrak{g}_{1} \neq\{0\}$.

Then $\mathfrak{s}$ is necessary identical to $\mathfrak{g}$.

Proof. We put $\mathfrak{h}=\mathfrak{G} \cap\left(\mathfrak{g}_{0}+\mathfrak{g}_{1}\right) / \mathfrak{s} \cap \mathfrak{g}_{1}$. Then $\mathfrak{h}$ can be considered as a subalgebra of $\mathfrak{g}_{0}$ satisfying the condition of Lemma (3-7). Therefore

$$
\mathfrak{S} \cap\left(\mathfrak{g}_{0} \oplus \mathfrak{g}_{1}\right) / \mathfrak{G} \cap \mathfrak{g}_{1}=\mathfrak{g}_{0} \quad \text { and } \quad \mathfrak{S} \cap \mathfrak{g}_{1}=\mathfrak{g}_{1} \text {. }
$$

Thus we have $\mathfrak{s} \supset \mathfrak{g}_{0}$. Hence $\mathfrak{B}=\mathfrak{g}$ by the condition (1).

4. Harmonic theory in the Spencer cohomologies (cf. [16]). Let $\mathfrak{g}=\mathfrak{g}_{-1} \oplus \mathfrak{g}_{0}$ $\oplus \mathfrak{g}_{1}$ be a semisimple graded Lie algebra over $\boldsymbol{F}$. We define a formal adjoint operator $\partial^{*}: C(\mathrm{~g}) \rightarrow C(\mathrm{~g})$ of $\partial$ to be $\partial^{*} C^{p, q}(\mathrm{~g}) \rightarrow C^{p+1, q-1}(\mathrm{~g})$, and

(4-1). $\left(\partial^{*} c\right)\left(x_{1}, \ldots, x_{q-1}\right)=\sum_{i}\left[Z^{i}, c\left(X_{i}, x_{1}, \ldots, x_{q-1}\right)\right]$ for all $c \in C^{p, q}(\mathfrak{g})$ and $x_{1}, \ldots, x_{q-1} \in \mathfrak{g}_{-1}$, where $\left\{X_{i}\right\}$ is a basis of $\mathfrak{g}_{-1}$ and $\left\{Z^{i}\right\}$ is the dual basis of $\left\{X_{i}\right\}$ in $\mathrm{g}_{1}$ (cf. Lemma (3-2) and Lemma (3-3)). It is evident that $\partial^{*}$ is well defined (i.e., independent of the choice of the basis $\left\{X_{i}\right\}$ ).

Lemma (4-1). We have $\partial^{*}(\rho(a) c)=\rho(a) \partial^{*} c$ for $c \in C(\mathfrak{g})$ and $a \in \mathfrak{g}_{0}$, here $\rho$ is the natural representation of $\mathrm{g}_{0}$ into $C(\mathrm{~g})$.

Proof. This is easy to see (cf. Lemma (3-3)).

We define the Laplacian $\square$ by $\square=\partial^{*} \partial+\partial \partial^{*}$. Then $\square$ is a linear endomorphism of $C^{p, q}(\mathrm{~g})$ for each $p, q$. Then the kernel of $\square: C^{p, q}(\mathfrak{g}) \rightarrow C^{p, q}(\mathrm{~g})$ is denoted by $\mathscr{H}^{p, q}(\mathfrak{g})\left(^{3}\right)$ and an element of $\mathscr{H}^{p, q}(\mathfrak{g})$ is called a harmonic form.

Lemma (4-2). We have $\rho(a) \square c=\square(\rho(a) c)$ for $c \in C(\mathfrak{g})$.

Proof. This follows from Lemma (2-5) and Lemma (4-1).

Proposition (4-1). We have

(4-2). $\partial C^{p, q}(\mathrm{~g}) \cap \mathscr{H}^{p-1, q+1}(\mathrm{~g})=\{0\}$,

(4-3). $H^{p, q}(\mathrm{~g}) \cong \mathscr{H}^{p, q}(\mathrm{~g})$.

Proof. To prove this it suffices to show the existence of an inner product (resp. hermitian inner product) ( , ) on $C(\mathfrak{g})$ (according as $\boldsymbol{F}=\boldsymbol{R}$ or $\boldsymbol{F}=\boldsymbol{C}$ ) such that $\partial^{*}$ is the adjoint operator with respect to the inner product $($,$) i.e., \left(\partial c, c^{\prime}\right)=\left(c, \partial^{*} c\right)$ for $c, c^{\prime} \in C(\mathrm{~g})[16]$. For that purpose we make use of the bilinear forms $B^{*}$ in Proposition (3-5). We define an inner product (resp. hermitian inner product) in $C(g)$ by

(4-4). $\left(c, c^{\prime}\right)=(1 / q !) \sum B^{*}\left(c\left(X_{i_{1}}, \ldots, X_{i_{q}}\right), c^{\prime}\left(X_{i_{1}}, \ldots, X_{i_{q}}\right)\right)$ for all $c, c^{\prime} \in C^{p, q}(\mathrm{~g})$, where $X_{i}$ is an orthonormal basis of $g_{-1}$ with respect to $B^{*}$. We put $\left(c, c^{\prime}\right)=0$ for $c \in C^{p, q}(\mathfrak{g}), c^{\prime} \in C^{p^{\prime}, q^{\prime}}(\mathrm{g})\left(p \neq p^{\prime}\right.$ or $\left.q \neq q^{\prime}\right)$. Now we prove

$\left.{ }^{3}\right)$ We should write $\mathscr{H}^{p, q}\left(\sum \mathfrak{g}_{p}\right)$, but we adopt this notation if there is no danger of confusion. 
(4-5). $\left(\partial c, c^{\prime}\right)=\left(c, \partial^{*} c^{\prime}\right)$ for $c, c^{\prime} \in C(\mathrm{~g})$. In fact, for $c \in C^{p, q}(\mathrm{~g})$ and $c^{\prime} \in C^{p-1, q+1}(\mathrm{~g})$, we get

$$
\begin{aligned}
\left(\partial c, c^{\prime}\right) & =-\frac{1}{(q+1) !} \sum B\left((\partial c)\left(X_{i_{1}}, \ldots, X_{i_{q+1}}\right), \sigma \cdot c^{\prime}\left(X_{i_{1}}, \ldots, X_{i_{q+1}}\right)\right) \\
& =-\frac{1}{(q+1) !} \sum \sum(-1)^{j} B\left(\left[c\left(X_{i_{1}}, \ldots, \hat{X}_{i_{j}}, \ldots, X_{i_{q+1}}\right), X_{i_{j}}\right],\right. \\
& =-\frac{1}{(q+1) !} \sum \sum(-1)^{j} B\left(c\left(X_{i_{1}}, \ldots, \hat{X}_{i_{j}}, \ldots, X_{i_{q+1}}\right),\right. \\
& =\frac{1}{(q+1) !} \sum \sum B\left(c\left(X_{i_{1}}, \ldots, X_{i_{q+1}}\right)\right) \\
& =\left(c, \partial^{*} c^{\prime}\right) .
\end{aligned}
$$

Here we remark that $\left\{-\sigma X_{i}\right\}$ is the dual basis of $\left\{X_{i}\right\}$.

5. The Spencer cohomologies of complex simple graded Lie algebras (cf. [16], [17], [18], [19]). To obtain the Spencer cohomologies of semisimple graded Lie algebras over $\boldsymbol{F}$, we have only to obtain that of complex one (see Lemma (2-4)). Then by Lemma (2-3) and Proposition (3-2), it is sufficient to consider complex simple one.

In this section $\mathfrak{g}=\mathfrak{g}_{-1} \oplus \mathfrak{g}_{0} \oplus \mathfrak{g}_{1}$ denotes a complex simple graded Lie algebra. And $e$ is the elements of Lemma (3-4).

LEMMA (5-1). We have

(5-1). $\mathrm{g}_{0}=\mathrm{Ce} \oplus\left[\mathrm{g}_{0}, \mathrm{~g}_{0}\right]$,

(5-2). $\left[\mathfrak{g}_{0}, \mathfrak{g}_{0}\right]$ is a semisimple Lie algebra.

Proof. This follows from the fact that $g_{0}$ has an irreducible faithful representation (cf. Proposition (3-3)).

From now on we fix once for all a Cartan subalgebra $\mathfrak{h}$ of $\mathfrak{g}$ such that $\mathfrak{g}_{0} \supset \mathfrak{h} \ni \boldsymbol{e}$ (cf. Corollary (3-1)). We denote by $\Delta$ the set of nonzero roots of $g$ with respect to $\mathfrak{h}$. For $\alpha \in \Delta, \mathfrak{g}_{\alpha}$ denotes the eigenspace of $\alpha$. The restriction of the Killing form $B$ of $\mathfrak{g}$ onto $\mathfrak{h}$ is still nondegenerate. Therefore we define $H_{\alpha}$ for each $\alpha \in \Delta$ by $B\left(H_{\alpha}, H\right)=\alpha(H), H \in \mathfrak{h}$. Let $\mathfrak{h}_{0}$ be the real subspace of $\mathfrak{h}$ spanned by $\left\{H_{\alpha} \mid \alpha \in \Delta\right\}$. It is known that $\mathfrak{h}_{0}$ is characterized as the maximal real subspace of $\mathfrak{h}$ on which all $\alpha \in \Delta$ has real value. Thus we have $e \in \mathfrak{h}_{0}$. The restriction of $B$ onto $\mathfrak{h}_{0}$ is positive definite. Thus we introduce an inner product $($,$) in \mathfrak{h}_{0}^{*}$. Then $(\alpha, \beta)$ $=B\left(H_{\alpha}, H_{\beta}\right)(\alpha, \beta \in \Delta)$.

Since $\mathfrak{h}$ is contained in $\mathfrak{g}_{0}, \Delta$ is a disjoint union of three subsets $\Delta_{-1}, \Delta_{0}$ and $\Delta_{1}$ such that

$$
\mathfrak{g}_{-1}=\sum_{\alpha \in \Delta-1} \mathfrak{g}_{\alpha}, \quad \mathfrak{g}_{0}=\mathfrak{h}+\sum_{\beta \in \Delta_{0}} \mathfrak{g}_{\beta}, \quad \mathfrak{g}_{1}=\sum_{\gamma \in \Delta_{1}} \mathfrak{g}_{\gamma}
$$

Lemma (5-2). We have $\Delta_{-1}=-\Delta_{1}$. 
Proof. This follows from Lemma (3-3).

Let $\mathfrak{g}_{u}$ be a real compact form of $\mathfrak{g}$ such that $\mathfrak{g}_{u} \cap \mathfrak{h}=\sqrt{ }-1 \mathfrak{h}_{0}$. Let $\sigma$ be the conjugation of $\mathfrak{g}$ with respect to $g_{u}$. Then $\sigma\left(H_{\alpha}\right)=-H_{\alpha}$ for all $\alpha \in \Delta$. Thus we have $\sigma\left(\mathfrak{g}_{\alpha}\right)=\mathfrak{g}_{-\alpha}$. Therefore we have

$$
\sigma\left(\mathfrak{g}_{-1}\right)=\mathfrak{g}_{1}, \quad \sigma\left(\mathfrak{g}_{0}\right)=\mathfrak{g}_{0}, \quad \sigma\left(\mathfrak{g}_{1}\right)=\mathfrak{g}_{-1} .
$$

Remark (5-1). Since the bilinear form $B^{*}$ defined by $B^{*}(x, y)=-B(x, \sigma(y))$ is hermitian symmetric, our $\sigma$ satisfies the conditions in Proposition (3-5).

LEMmA (5-3). For $\alpha \in \Delta_{-1} \cup \Delta_{1}$, we can choose a root vector $X_{\alpha} \in \mathfrak{g}_{\alpha}$ such that

(i) $\left\{X_{\alpha}\right\}_{\alpha \in \Delta_{-1}}$ is an orthonormal basis of $\mathfrak{g}_{-1}$ with respect to $B^{*}$.

(ii) $\left\{X_{-\alpha}\right\}_{\alpha \in \Delta_{-1}}$ is the dual basis of $\left\{X_{\alpha}\right\}_{\alpha \in \Delta_{-1}}$ in $\mathrm{g}_{1}$ with respect to $B$.

(iii) $\sigma\left(X_{\alpha}\right)=-X_{-\alpha}$.

(iv) $\left[X_{\alpha}, X_{-\alpha}\right]=H_{\alpha}$.

Proof. This follows from the fact that for $\alpha, \beta \in \Delta_{-1} \cup \Delta_{1}, B\left(X_{\alpha}, X_{\beta}\right)=0$ unless $\alpha+\beta=0,\left[X_{\alpha}, X_{-\alpha}\right]=B\left(X_{\alpha}, X_{-\alpha}\right) H_{\alpha}$, and $\sigma\left(g_{\alpha}\right)=\mathfrak{g}_{-\alpha}$.

For the matter of convenience, we denote by $\left\{\alpha_{1}, \ldots, \alpha_{N}\right\}$ be the element in $\Delta_{1}$. We write $X_{i}$ and $X_{i}^{-}$instead of $X_{\alpha_{i}}$ and $X_{-\alpha_{i}}$ respectively. Since $\sigma\left(g_{0}\right)=g_{0}$, we can choose a basis $\left\{Y_{a}\right\}_{a=1, \ldots, r}$ of $g_{0}$ such that $B\left(Y_{a}, Y_{b}\right)=-\delta_{a b}$. We fix once for all those $X_{i}, Y_{a}$. Then it is easy to see that Laplacian $\square: C^{p, q}(\mathrm{~g}) \rightarrow C^{p, q}(\mathrm{~g})$ is expressed by

$$
\begin{aligned}
(\square c)\left(X_{i_{1}}, \ldots, X_{i_{q}}\right)= & \sum_{k=1}^{N}\left[X_{k}\left[X_{\bar{k}} c\left(X_{i_{1}}, \ldots, X_{i_{q}}\right)\right]\right] \\
& +\sum_{k=1}^{N} \sum_{u=1}^{q}(-1)^{u+1}\left[\left[X_{i_{u}}, X_{k}\right] c\left(X_{\bar{k}}, X_{i_{1}}, \ldots, \hat{X}_{i_{u}}, \ldots, X_{i_{q}}\right)\right] .
\end{aligned}
$$

For an $x \in \mathfrak{g}_{1}, \varepsilon(x)$ is the operator of exterior multiplication by $x$ on $\Lambda g_{1}$, and for $X_{k}^{0}, i\left(X_{k}\right)$ is the operator on $\Lambda g_{1}$ defined as usual by the formula

$$
i\left(X_{k}\right)\left(z_{1} \cdots z_{p}\right)=\sum_{u=1}^{p}(-1)^{u}\left\langle X_{k}, z_{u}\right\rangle z_{1} \wedge \cdots \wedge \hat{z}_{u} \wedge \cdots \wedge z_{p}
$$

We denote by ad ${ }_{+}\left(\right.$resp. $\left.\operatorname{ad}_{+}^{q}\right)$ the representation of $g_{0}$ into $\Lambda_{\mathfrak{g}_{1}}$ (resp. $\left.\Lambda^{q} \mathfrak{g}_{1}\right)$.

LEMMA (5-4). We have

$$
\operatorname{ad}_{+}\left(Y_{a}\right)=\sum_{k=1}^{N} \varepsilon\left(\left[Y_{a}, X_{k}\right]\right) i\left(X_{k}\right)
$$

Proof. It suffices to show this on $\Lambda^{1} \mathfrak{g}_{1}=\mathfrak{g}_{1}$.

Now for $X_{j} \in g_{1}$,

$$
\left(\sum_{k=1}^{N} \varepsilon\left(\left[Y_{a}, X_{k}\right]\right) i\left(X_{k}\right)\right)\left(X_{j}\right)=\sum_{k=1}^{N} B\left(X_{k}, X_{j}\right)\left[Y_{a}, X_{k}\right]=\left[Y_{a}, X_{j}\right]=\left(\operatorname{ad}_{+} Y_{a}\right)\left(X_{j}\right)
$$

We put $\left[X_{i}, X_{j}\right]=\sum C_{a i j} Y_{a}(i, j=1, \ldots, N)$. From the relation

$$
B\left(\left[X_{i}, Y_{a}\right], X_{j}\right)+B\left(Y_{a},\left[X_{i}, X_{j}\right]\right)=0,
$$

we get $\left[Y_{a}, X_{i}\right]-=\sum_{j=1}^{N} C_{a i j} X_{j}$. 
We use the following two nontrivial lemmas without proof (see [17]).

LEMMA (5-5). We have

(5-5). $e=2 \sum_{\alpha \in \Delta_{1}} H_{\alpha}$,

(5-6). $\sum_{a=1}^{r}\left(\operatorname{ad}_{+}^{q}\left(Y_{a}\right)\right)^{2}=-\sum_{k=1}^{N} \operatorname{ad}_{+}^{q}\left(\left[X_{k}, X_{\bar{k}}\right]\right)=-(q / 2) 1$ on $\Lambda^{q} \mathfrak{g}_{1}$,

here 1 denotes the identity map.

Since $\mathfrak{g}_{-1}$ and $\mathfrak{g}_{1}$ are dual with each other, we can identify $C^{p, q}(\mathfrak{g})=\mathfrak{g}_{p-1} \otimes$ $\Lambda^{q}\left(\mathfrak{g}_{-1}\right)^{*}$ with $\mathfrak{g}_{p-1} \otimes \Lambda^{q} \mathfrak{g}_{1}$ by

$$
c \in C^{p, q}(g) \mapsto \frac{1}{q !} \sum c\left(X_{i_{1}}, \ldots, X_{i_{q}}\right) X_{i_{1}} \wedge \cdots \wedge X_{i_{q}} .
$$

Then the Laplacian $\square$ can be expressed on $\mathfrak{g} \otimes \Lambda \mathfrak{g}_{1}$ by

$$
\square=\sum_{k=1}^{N} \operatorname{ad} X_{k} \cdot \operatorname{ad} X_{k} \otimes 1+\sum_{k, u=1}^{N} \operatorname{ad}\left[X_{\bar{u}}, X_{k}\right] \otimes \varepsilon\left(X_{u}\right) i\left(X_{k}\right) .
$$

Lemma (5-6). The Laplacian $\square$ has the following expression on $\mathfrak{g} \otimes \Lambda \mathfrak{g}_{1}$ :

$$
\square=\sum_{k=1}^{N} \operatorname{ad}\left(X_{k}\right) \operatorname{ad}\left(X_{k}\right) \otimes 1+\sum_{a=1}^{r} \operatorname{ad}\left(Y_{a}\right) \otimes \operatorname{ad}_{+}\left(Y_{a}\right)
$$

Proof.

$$
\begin{aligned}
& \sum_{k, u=1}^{N} \operatorname{ad}\left[X_{\bar{u}}, X_{k}\right] \otimes \varepsilon\left(X_{u}\right) i\left(X_{k}\right) \\
& \quad=-\sum_{a, k, u} C_{\alpha k \bar{u}} \operatorname{ad}\left(Y_{a}\right) \otimes \varepsilon\left(X_{u}\right) i\left(X_{k}\right) \quad \text { (see the remark after Lemma (5-4)) } \\
& \quad=-\sum_{a, k, u} \operatorname{ad}\left(Y_{a}\right) \otimes \varepsilon\left(C_{\alpha k \bar{u}} X_{u}\right) i\left(X_{k}\right) \\
& \quad=\sum_{a, k} \operatorname{ad}\left(Y_{a}\right) \otimes \varepsilon\left[Y_{a}, Y_{k}\right] i\left(X_{k}\right)=\sum_{a} \operatorname{ad} Y_{a} \otimes \mathrm{ad}_{+}\left(Y_{a}\right) \quad \text { (by Lemma (5-4)) }
\end{aligned}
$$

Let now $c$ be the element of the universal enveloping algebra $\mathscr{E}$ of $g$ defined by

$$
c=\sum_{k=1}^{N}\left(X_{k} X_{k}+X_{k} X_{k}\right)-\sum_{a=1}^{r} Y_{a}^{2} .
$$

The element $c$ is called the Casimir element of $\mathscr{E}$ and is in the center of $\mathscr{E}$. We put $c^{\prime}=-\sum_{a} Y_{a}^{2}$. Then $c^{\prime}$ is an element in the center of the enveloping algebra $\mathscr{E}^{\prime}$ of $g_{0}$. Extending the representation of $\mathfrak{g}$ (resp. $\mathfrak{g}_{0}$ ) to those of $\mathscr{E}$ (resp. $\mathscr{E}^{\prime}$ ), we get the following formula.

LEMMA (5-7). The Laplacian $\square$ has the following expression on $\mathfrak{g}$.

$$
2 \square=\operatorname{ad}(c) \otimes 1-\left(\operatorname{ad} \otimes \operatorname{ad}_{+}\right)\left(c^{\prime}\right)+\frac{1}{2}\left(\operatorname{ad} \otimes \operatorname{ad}_{+}\right)(e) .
$$

Here $\mathrm{ad} \otimes \mathrm{ad}_{+}$denotes the product of two representations ad and $\mathrm{ad}+\cdot$ 
Proof. $2 \sum \operatorname{ad}\left(X_{k}\right)$ ad $\left(X_{k}\right)=\operatorname{ad}(c)-\operatorname{ad}\left(c^{\prime}\right)+\sum \operatorname{ad}\left(\left[X_{k}, X_{k}\right]\right)$. On the other hand $\left(\operatorname{ad} \otimes \operatorname{ad}_{+}\right)\left(Y_{a}\right)=\operatorname{ad}\left(Y_{a}\right) \otimes 1+1 \otimes \operatorname{ad}_{+}\left(Y_{a}\right)$. Thus

$\left(\left(\operatorname{ad} \otimes \operatorname{ad}_{+}\right)\left(Y_{a}\right)\right)^{2}=\left(\operatorname{ad} Y_{a}\right)^{2} \otimes 1+1 \otimes\left(\operatorname{ad}_{+}\left(Y_{a}\right)\right)^{2}+2 \operatorname{ad}\left(Y_{a}\right) \otimes \operatorname{ad}_{+}\left(Y_{a}\right)$.

Hence

$2 \sum \operatorname{ad}\left(Y_{a}\right) \otimes \operatorname{ad}_{+}\left(Y_{a}\right)$

$$
\begin{aligned}
& =\sum\left(\left(\operatorname{ad} \otimes \operatorname{ad}_{+}\right)\left(Y_{a}\right)\right)^{2}-\sum\left(\operatorname{ad}\left(Y_{a}\right)\right)^{2} \otimes 1-\sum 1 \otimes\left(\operatorname{ad}_{+}\left(Y_{a}\right)\right)^{2} \\
& =-\left(\operatorname{ad} \otimes \operatorname{ad}_{+}\right)\left(c^{\prime}\right)+\operatorname{ad}\left(c^{\prime}\right) \otimes 1+\sum 1 \otimes \operatorname{ad}_{+}\left[X_{k}, X_{k}\right]
\end{aligned}
$$

(see Lemma (5-5)). From this we have a desired expression.

Now we choose, once and for all, an ordering of the roots such that the roots belonging to $\Delta_{1}$ are all positive. The existence of such an order is well known. Let $\Theta$ be the set of all positive roots $\alpha$ of $\mathfrak{g}$ such that $\mathfrak{g}_{\alpha} \subset \mathfrak{g}_{0}$. Then $\Delta^{+}=\Delta_{1} \cup \Theta$ is the set of all positive roots. We denote by $\left\{\gamma_{1}, \ldots, \gamma_{0}\right\}$ the set of all simple roots. Then we know that we may assume $\gamma_{1} \in \Delta_{1}$ and $\gamma_{2}, \ldots, \gamma_{l} \in \Theta$ [9]. Then $\gamma_{2}, \ldots, \gamma_{l}$ are also simple as the roots of the complex reductive Lie algebra $g_{0}$. Under these notations, if $\rho$ (resp. $\tau$ ) is an irreducible representation of $g$ (resp. $g_{0}$ ) with the lowest weight $\Lambda^{\prime}$ (resp. $\mu^{\prime}$ ), then $\rho(c)$ and $\tau\left(c^{\prime}\right)$ are scalar operators which is known to be given by

(5-9). $\rho(c)=\left\{\left(\Lambda^{\prime}, \Lambda^{\prime}\right)-\sum_{\alpha \in \Delta^{+}}\left(\Lambda^{\prime}, \alpha\right)\right\} \cdot 1$,

(5-10). $\tau\left(c^{\prime}\right)=\left\{\left(\mu^{\prime}, \mu^{\prime}\right)-\sum_{\beta \in \Theta}\left(\mu^{\prime}, \beta\right)\right\} \cdot 1$.

Let

$$
\mathfrak{g} \otimes \Lambda \mathfrak{g}_{1}=\sum n_{\xi^{\prime}}, U_{\xi^{\prime}}
$$

be a decomposition of the $\mathfrak{g}_{0}$-module $\mathfrak{g} \otimes \Lambda \mathfrak{g}_{1}$ into direct sum of irreducible $\mathfrak{g}_{0^{-}}$ modules $U_{\xi^{\prime}}$ where $\xi^{\prime}$ denotes the lowest weight of the irreducible representation $\tau_{\xi^{\prime}}$ of $g_{0}$ into $U_{\xi^{\prime}}$. Since $e$ is in the center $g_{0}$, the last term in the expression (5-8) is a scalar operator on each $U_{\xi^{\prime}}$. We denote by $\Lambda^{\prime}$ the lowest root of $\mathfrak{g}$ and put $\delta=\frac{1}{2} \sum_{\alpha \in \Delta}+\alpha$.

Proposition (5-1) (B. Kostant [16]). We have

$$
\square \mid U_{\xi^{\prime}}=\left\{\left(\Lambda^{\prime}-\delta, \Lambda^{\prime}-\delta\right)-\left(\xi^{\prime}-\delta, \xi^{\prime}-\delta\right)\right\} \cdot 1 .
$$

Proof. This follows from (5-8), (5-9), (5-10) and the above remark.

COROLlARY (5-1). Let $\mathfrak{g} \otimes \Lambda \mathfrak{g}_{1}=\sum n_{\xi^{\prime}}, U_{\xi^{\prime}}$ be as above. Then we have

$$
\mathscr{H}(\mathrm{g}) \cong \sum_{\left|\Lambda^{\prime}-\delta\right|=\left|\xi^{\prime}-\delta\right|} n_{\xi^{\prime}} U_{\xi^{\prime}}
$$

Here $\left|\Lambda^{\prime}-\delta\right|^{2}=\left(\Lambda^{\prime}-\delta, \Lambda^{\prime}-\delta\right)$.

Proof. This follows from proposal (5-1) and Lemma (4-2).

For a subset $\Phi$ of $\Delta$, we denote by $\langle\Phi\rangle$ the sum of the roots belonging to $\Phi$. 
Let $W$ be the Weyl group of $g$. And for an element $T$ of $W$, we put $\Phi_{T}=T\left(-\Delta^{+}\right)$ $\cap \Delta^{+}$. Put $W^{1}=\left\{T \in W \mid \Phi_{T} \subset \Delta_{1}\right\}$. Let $\zeta^{\prime}$ be the lowest weight of an irreducible representation $\rho_{\zeta^{\prime}}$ of $\mathfrak{g}$ with the representation space $V$. And let

$$
V \otimes \Lambda \mathfrak{g}_{1}=\sum l_{\xi^{\prime}} U_{\xi^{\prime}}
$$

be a decomposition of the $g_{0}$-module $V \otimes \Lambda g_{1}$ into direct sum of irreducible go-module $U_{\xi^{\prime}}$. For $T \in W^{1}$, we put $\zeta_{T}^{\prime}=T_{\zeta^{\prime}}+\left\langle\Phi_{T}\right\rangle$. Clearly $\zeta_{T}^{\prime}$ is a weight of the $\mathrm{g}_{0}$-module $V \otimes \Lambda \mathrm{g}_{1}$.

LEMMA (5-8). We have

(1) Let $\eta$ be a weight of the $\mathrm{g}_{0}$-module $V \otimes \Lambda \mathrm{g}_{1}$, then $\left|\Lambda^{\prime}-\delta\right| \geqq|\eta-\delta|$ and the equality holds if and only if there exists $T \in W^{1}$ such that $\eta=\zeta_{T}^{\prime}$.

(2) The map $T \mapsto \zeta_{T}^{\prime}$ is a bijection of $W^{1}$ into the set of the lowest weight $\xi^{\prime}$ appearing in the decomposition (5-13) and satisfying the equality $\left|\Lambda^{\prime}-\delta\right|=\left|\xi^{\prime}-\delta\right|$.

Proof. See [19].

Proposition (5-2) (B. Kostant [16]). We have $\mathscr{H}(\mathrm{g})=\sum_{T \in W^{1}} U_{\Lambda_{T}^{\prime}}$. Here $\Lambda^{\prime}$ is the lowest root of $\mathrm{g}$ and $\Lambda_{T}^{\prime}=T \Lambda^{\prime}+\left\langle\Phi_{T}\right\rangle$.

Proof. This follows from Corollary (5-1) and Lemma (5-8).

Let $n(T)$ denote the number of the root belonging to $\Phi_{T}$.

Corollary (5-2). We have $\sum_{p} \mathscr{H}^{p, q}(\mathrm{~g})=\sum_{T \in W^{1}, n(T)=q} U_{\Lambda^{\prime} T}$.

Proposition (5-3) (B. Kostant [16]). The $\mathfrak{g}_{0}$-module $\Lambda \mathfrak{g}_{1}$ and $\Lambda^{q} \mathfrak{g}_{1}$ decompose into a direct sum of $\mathrm{g}_{0}$-irreducible modules

$$
\Lambda \mathfrak{g}_{1}=\sum_{T \in W^{1}} U_{\left\langle\Phi_{T}\right\rangle}, \quad \Lambda^{q} \mathfrak{g}_{1}=\sum_{T \in W^{1}, n(T)=q} U_{\left\langle\Phi_{T}\right\rangle}
$$

Proof. This follows from Lemma (5-5) and Lemma (5-8).

Let $\mathfrak{g}=\sum m_{\xi^{\prime}} \mathfrak{g}_{\xi^{\prime}}$ be a decomposition of the $\mathfrak{g}_{0}$-module $\mathfrak{g}$ into direct sum of irreducible $g_{0}$-module $g_{\xi^{\prime}}$ with the lowest weight $\xi^{\prime}$. Combining this with the decomposition $\Lambda g_{1}=\sum_{T \in W^{1}} U_{\left\langle\Phi_{T}\right\rangle}$, we have

$$
\mathfrak{g} \otimes \Lambda \mathfrak{g}_{1}=\sum_{\xi^{\prime}} \sum_{T \in W^{1}} m_{\xi^{\prime}} \mathfrak{g}_{\xi^{\prime}} \otimes U_{\left\langle\Phi_{T}\right\rangle}
$$

Lemma (5-9). Suppose that there exists an element $c \in \mathfrak{g}_{\xi^{\prime}} \otimes U_{\left\langle\Phi_{T}\right\rangle}$ such that $c \neq 0$ and $\square c=0$. Then $\xi^{\prime}=T \Lambda^{\prime}$.

Proof [19]. By the assumption, the intersection of $g_{\xi^{\prime}} \otimes U_{\left\langle\Phi_{T}\right\rangle}$ with $\sum_{T \in W^{1}} U_{\Lambda_{T}^{\prime}}$ is not empty. Therefore there exists $S \in W^{1}$ such that $U_{\Lambda_{s}^{\prime}}$ is contained in $g_{\xi^{\prime}} \otimes$ $U_{\left\langle\Phi_{T}\right\rangle}$. Then $\Lambda_{S}^{\prime}$ is a weight of the $g_{0}$-module $g_{\xi^{\prime}} \otimes U_{\left\langle\Phi_{T}\right\rangle}$ and hence there exists a weight $\nu$ in $\mathfrak{g}_{\xi^{\prime}}$ and a subset $\Phi$ of $\Delta_{1}$ such that $\Lambda_{S}^{\prime}=\nu+\langle\Phi\rangle$. Then by Lemma (5-8) we get $\nu=S \Lambda^{\prime}$ and $\Phi=\Phi_{S}$. We remark now that $R^{-1}(\Theta) \subset \Theta$ for an $R \in W^{1}$. Therefore $\left(S \Lambda^{\prime}, \beta\right)=\left(\Lambda^{\prime}, S^{-1} \beta\right) \leqq 0$ for all $\beta \in \Theta$ because $\Lambda^{\prime}$ is the lowest root of 
$g_{0}$. It follows from this that $\nu=S \Lambda^{\prime}$ is the lowest weight in $\mathfrak{g}_{\xi^{\prime}}$ and hence $\xi^{\prime}=S \Lambda^{\prime}$. On the other hand, since $\left\langle\Phi_{S}\right\rangle$ is the lowest weight in $U_{\left\langle\Phi_{S}\right\rangle}$, we have $\left(\left\langle\Phi_{S}\right\rangle, \beta\right) \leqq 0$ for $\beta \in \Theta$. Therefore, as $\left\langle\Phi_{S}\right\rangle$ is a weight in $U_{\left\langle\Phi_{T}\right\rangle}$, we get $\left\langle\Phi_{T}\right\rangle=\left\langle\Phi_{S}\right\rangle$. Then by the lemma, we get $T=S$.

Corollary (5-3) (MATSushima-Murakami [18]). Let $q_{\text {ad }}$ be the numbers of the roots in $\left\{\alpha \in \Delta_{1}\left|\left\langle\alpha, \mu^{\prime}\right\rangle\right\rangle 0\right\}$, here $\mu^{\prime}$ is the lowest root in $\Delta_{1}$. Then we have

$$
H^{2, q}(\mathfrak{g})=0 \quad \text { if } q<q_{\mathrm{ad}} .
$$

Proof. By Proposition (4-1) and Corollary (5-2), we have

$$
H^{2, q}(\mathfrak{g})=\mathscr{H}^{2, q}(\mathrm{~g})=\sum_{p} \mathscr{H}^{p, q}(\mathfrak{g}) \cap \mathfrak{g}_{1} \otimes \Lambda \mathfrak{g}_{1}
$$

By Lemma (5-9), $\sum_{p} \mathscr{H}^{p, q}(\mathfrak{g}) \cap g_{1} \otimes U_{\left\langle\Phi_{T}\right\rangle} \neq 0$ only if $\mu^{\prime}=T \Lambda^{\prime}$ and $n(T)=q$. If $\left(\alpha, \mu^{\prime}\right)>0\left(\alpha \in \Delta_{1}\right)$, then $0<\left(\alpha, \mu^{\prime}\right)=\left(\alpha, T \Lambda^{\prime}\right)=\left(T^{-1} \alpha, \Lambda^{\prime}\right)$. Since $\Lambda^{\prime}$ is the lowest weight, $T^{-1} \alpha<0$, i.e., $\alpha \in \Phi_{T}$. Thus $q_{\text {ad }} \leqq n(T)$.

LeMmA (5-10). If $H^{1,1}(\mathrm{~g}) \neq 0$, then $\mathrm{g}_{0}$ has an element of rank $1, \mathrm{~g}_{0}$ being considered as the subalgebra of $\mathfrak{g l}\left(\mathfrak{g}_{-1}\right)$.

Proof. Suppose $H^{1,1}(\mathrm{~g}) \neq 0$. Then $\mathrm{g}_{0} \otimes \mathrm{g}_{1}$ contains $U_{\Lambda_{T}^{\prime}}$ for some $T \in W^{1}$. Taking an eigenvector of the lowest weight $\Lambda_{T}^{\prime}=T \Lambda^{\prime}+\left\langle\Phi_{T}\right\rangle$, we can assume $\mathscr{H}^{1,1}(\mathrm{~g})$ contains an element of the form $y \otimes z\left(y \in \mathfrak{g}_{0}, z \in \mathfrak{g}_{1}\right)$. Since $\partial(y \otimes z)=0$, for $x, x^{\prime} \in g_{-1}$, we have

$$
B(x, z)\left[y, x^{\prime}\right]=B\left(x^{\prime}, z\right)[y, x] .
$$

Taking $x^{\prime}$ as $B(x, z)=1$, we have $[y, x]=B(x, z)\left[y, x^{\prime}\right]$, i.e., $y$ is an element of rank 1 .

RemarK. Simpler proof may be obtained using Guillemin, Quillen, Sternberg, Classification of the irreducible algebras of infinity type, J. Analyse Math. 23 (1967).

6. Semisimple homogeneous spaces. Let $L / L_{0}$ be a connected homogeneous space on which a semisimple Lie group $L$ acts effectively and transitively. Here $L$ is not necessary connected. $L / L_{0}$ is called a semisimple flat homogeneous space if the Lie algebra $\mathfrak{l}$ of $L$ has a graded Lie algebra structure $\mathfrak{l}=\mathfrak{g}_{-1} \oplus \mathfrak{g}_{0} \oplus \mathfrak{g}_{1}$ such that $\mathfrak{g}_{0} \oplus \mathfrak{g}_{1}$ is the Lie algebra of $L_{0}$. We call the graded Lie algebra $\mathfrak{l}=\mathfrak{g}_{-1} \oplus \mathfrak{g}_{0}$ $\oplus \mathfrak{g}_{1}$ the associated graded Lie algebra of a semisimple homogeneous space $L / L_{0}$ (cf. Proposition (3-4)). Let $G_{1}$ (resp. $G_{-1}$ ) be the connected Lie subgroup of $L$ corresponding to $\mathfrak{g}_{1}$ (resp. $\mathfrak{g}_{-1}$ ). We define $G_{0}$ as the normalizer of $\mathfrak{g}_{0}$ in $L_{0}$, i.e., $G_{0}=\left\{a \in L_{0} \mid \operatorname{Ad}(a)\left(g_{0}\right)=g_{0}\right\}$.

Lemma (6-1). The exponential mapping exp: $\mathfrak{g}_{1} \rightarrow G_{1}$ and exp: $\mathfrak{g}_{-1} \rightarrow G_{-1}$ are bijective.

Proof. Since $\mathfrak{g}_{1}$ is abelian, exp is surjective. Let $\mathfrak{n}$ be the kernel of exp: $\mathfrak{g}_{1} \rightarrow G_{1}$. Since $\mathfrak{n}$ is discrete we have $\left[g_{0}, \mathfrak{n}\right]=0$. This is contradiction (see Lemma (3-4)). 
LEMMA (6-2). $G_{0}$ is identical to the normalizer of $\mathrm{g}_{-1}$ in $L_{0}$, i.e.,

$$
G_{0}=\left\{a \in L_{0} \mid \operatorname{Ad}(a)\left(\mathfrak{g}_{-1}\right)=\mathfrak{g}_{-1}\right\} .
$$

Proof. Let $G_{0}^{\prime}$ be $\left\{a \in L_{0} \mid \operatorname{Ad}(a)\left(g_{-1}\right)=g_{-1}\right\}$. Take an element $a$ in $G_{0}$. Since Ad $(a)\left(g_{0}\right)=\mathfrak{g}_{0}$ and Ad $(a)\left(g_{1}\right)=\mathfrak{g}_{1},\left(\mathfrak{g}_{1}=\left\{x \in \mathfrak{g}_{0} \oplus \mathfrak{g}_{1} \mid[\mathfrak{g}, x] \subset \mathfrak{g}_{0}\right\}\right)$ by the argument in Proposition (3-4), there exists an isomorphism $\phi$ of $\mathfrak{g}$ such that $\phi\left(\mathfrak{g}_{-1}\right)=\mathfrak{g}_{-1}$ and $\phi\left|\mathfrak{g}_{0}+\mathfrak{g}_{1}=\operatorname{Ad}(a)\right| g_{0}+\mathfrak{g}_{1}$. Then $\phi \cdot \operatorname{Ad}(a)^{-1}$ is, in particular, the identity on $\mathfrak{g}_{0}$. Since $\mathfrak{g}_{0}$ contains a Cartan subalgebra $\mathfrak{h}$ of $\mathfrak{g}$, there exists an element $H$ of $\mathfrak{h}$, such that $\phi \cdot \operatorname{Ad}(a)^{-1}=\exp$ ad $H$. From this we have $\operatorname{Ad}(a)\left(\mathfrak{g}_{-1}\right)=\mathfrak{g}_{-1}$. Take $a \in G_{0}^{\prime}$, then

$$
\operatorname{Ad}(a)\left(\mathfrak{g}_{-1} \oplus \mathfrak{g}_{1}\right)=\mathfrak{g}_{-1} \oplus \mathfrak{g}_{1}
$$

Since $g_{0}$ is the orthogonal complement of $\mathfrak{g}_{-1} \oplus \mathfrak{g}_{1}$ with respect to the Killing form $B$, thus $\operatorname{Ad}(a)\left(g_{0}\right)=g_{0}$ since $\operatorname{Ad}(a)$ leaves $B$ invariant.

Lemma (6-3). We have $G_{0} \cap G_{1}=\{\mathrm{id}\}$.

Proof. Let $Z \in g_{1}$ be such that $\exp Z \in G_{0} \cap G_{1}$. For $X \in \mathfrak{g}_{-1}$,

$$
\operatorname{Ad}(\exp Z)(X)=X+[Z, X]+\frac{1}{2}[Z[Z, X]] \text {. }
$$

Since $\operatorname{Ad}(\exp Z)\left(\mathfrak{g}_{-1}\right) \subset \mathfrak{g}_{-1}$, we have $\left[Z, \mathfrak{g}_{-1}\right]=0$. This is a contradiction (see (2-3)).

Lemma (6-4). We have $L_{0}=G_{0} \cdot G_{1}$.

Proof. Let $c$ be the center of $g_{0}$. From Lemma (5-1) we can easily see that ad $c(c \in \mathfrak{c})$ is semisimple. From the argument in Proposition (3-4) for $a \in L_{0}$ there exists an element $Z$ in $\mathfrak{g}_{1}$ such that $\operatorname{Ad}(\exp Z) \operatorname{Ad}(a)$ preserves $\mathfrak{g}_{1}, \mathfrak{g}_{1}+\mathfrak{c}$ and $\left[g_{0}, \mathfrak{g}_{0}\right]$. From the above remark we see $\operatorname{Ad}(\exp Z) \operatorname{Ad}(a)(\mathfrak{c})=\mathfrak{c}$. Thus we have $\operatorname{Ad}(\exp Z) \operatorname{Ad}(a)\left(g_{0}\right)=g_{0}$. Then $\exp Z \cdot a \in G_{0}$ i.e., $L_{0}=G_{1} \cdot G_{0}=G_{0} \cdot G_{1}$.

COROLlaRY (6-1). $L_{0}$ is the semidirect product of $G_{0}$ and $G_{1}$.

We have a natural representation $l$ of $L_{0}$ into $\mathrm{g}_{-1}$ (so-called the linear isotropy representation of $L_{0}$ ). It is defined by

$$
l(a) x=\operatorname{Ad}(a) x \bmod \left(\mathrm{g}_{0} \oplus \mathrm{g}_{1}\right) \quad \text { for } a \in L_{0} .
$$

Lemma (6-5). $G_{1}$ is identical to the kernel of $l$, or equivalently $l$ is faithful on $G_{0}$.

Proof. It is clear that $G_{1}$ is contained in the kernel of $l$. Take $a \in G_{0}$ such that $\operatorname{Ad}(a) \mid g_{1}$ is the identity. Then by Lemma (3-3), Ad $(a) \mid g_{-1}$ is also the identity. Since $\mathfrak{g}_{0}=\left[\mathfrak{g}_{-1}, \mathfrak{g}_{1}\right]$, Ad $(a)$ is the identity on $\mathfrak{l}$, i.e., $a$ commutes with the identity connected component of $L$. This is a contradiction because the operation of $L$ is effective.

The group $G_{0}$ is called the linear isotropy subgroup of the semisimple flat homogeneous space $L / L_{0}$. 
7. Classification of simple graded Lie algebras. We can classify all semisimple graded Lie algebras over $\boldsymbol{F}(\boldsymbol{F}=\boldsymbol{R}$ or $\boldsymbol{C})$. By Proposition (3-2), we have only to classify simple graded Lie algebras. This has been done in [14]. And the value $q_{\text {ad }}$ (cf., Corollary (5-3)) for complex simple Lie algebras has been computed by A. Borel [1]. We here only write the table. For the details, see [14].

Let $K$ be the field $\boldsymbol{R}$ of real numbers, the field $\boldsymbol{C}$ of complex numbers or the field $\boldsymbol{Q}$ of quaternions. In a natural way, $\boldsymbol{R} \subset \boldsymbol{C} \subset \boldsymbol{Q}$. For each element $x$ of $K$, we define the elements $\bar{x}$ and $\tilde{x}$ as follows.

If $x=x_{0}+x_{1} i+x_{2} j+x_{3} k \in Q$ with $x_{0}, x_{1}, x_{2}, x_{3} \in R$. Then

$$
\bar{x}=x_{0}-x_{1} i-x_{2} j-x_{3} k, \quad \tilde{x}=x_{0}+x_{1} i-x_{2} j-x_{3} k .
$$

We use the following notations.

(1) $\operatorname{gl}(n ; K)=\{$ all $n \times n$ matrices over the field $K\}$.

(2) $\operatorname{sl}(n ; K)=$ the semisimple part of $\mathfrak{g l}(n ; K)$.

(3) $\mathfrak{S O}(p, q ; K)=\left\{A \in \mathfrak{g l}(p+q ; K) \mid{ }^{t} \tilde{A} I_{p, q}+I_{p, q} A=0\right\}$, where

$\mathfrak{S} \mathfrak{p}(n ; K)=\mathfrak{s} \mathfrak{p}(n, 0 ; K)$.

$$
I_{p, q}=\left(\begin{array}{cr}
I_{p} & 0 \\
0 & -I_{q}
\end{array}\right)
$$

(4) $\mathfrak{u}(p, q ; K)=\left\{A \in \mathfrak{g l}(p+q ; K) \mid{ }^{t} \bar{A} I_{p, q}+I_{p, q} A=0\right\}$. $\mathfrak{u}(n ; K)=\mathfrak{u}(n, 0 ; K)$.

(5) $\mathfrak{s} \mathfrak{u}(p, q ; K)=\mathfrak{u}(p, q ; K) \cap \mathfrak{s l}(p+q ; K)$.

(6) $\mathfrak{B p}(n ; K)=\left\{A \in \operatorname{gl}(z n ; K) \mid{ }^{t} \widetilde{A} J+J A=0\right\}$.

\begin{tabular}{|c|c|c|c|c|c|c|}
\hline ype & $\mathrm{I}_{p, q}(p \geqq q \geqq 1)$ & $\mathrm{II}_{p}(p \geqq 2)$ & $\operatorname{III}_{p}(p \geqq 2) \mid$ & $\mathrm{IV}_{\mathrm{p}}$ & V & VI \\
\hline $\mathfrak{g}$ & ;C) & $(2 p ; C)$ & $\mathfrak{S P}(n ; C)$ & $\mathfrak{S O}^{\mathfrak{O}}(p+q+2 ; C)$ & $E_{0}^{C}$ & $E_{7}^{C}$ \\
\hline$g_{0}$ & $\mathfrak{S l}(p ; \boldsymbol{C})+\mathfrak{B l}(q, \boldsymbol{C})+\boldsymbol{C}$ & $\mathfrak{g l}(p ; C)$ & $\mathfrak{g l}(n ; C)$ & $\mathfrak{s} \mathfrak{b}(p+q ; C)+C$ & $\mathfrak{S O}(10: C)+C$ & $E_{6}^{C+}$ \\
\hline$q_{\mathrm{ad}}$ & $p+q-1$ & $2 p-3$ & $p$ & $p+q-1$ & 11 & 17 \\
\hline
\end{tabular}

I. Complex simple graded Lie algebras $\mathfrak{g}=\mathfrak{g}_{-1}+\mathfrak{g}_{0}+\mathfrak{g}_{1}$.

II. Real simple graded Lie algebras $\mathfrak{g}=\mathfrak{g}_{-1}+\mathfrak{g}_{0}+\mathfrak{g}_{1}$.

\begin{tabular}{|c|c|c|c|}
\hline $\mathfrak{g}$ & $\mathfrak{S I}(p+q ; \boldsymbol{R})$ & $\mathfrak{B I}(p+q ; C)$ & $\mathfrak{S} \mathfrak{u}^{*}(2 p+2 q)$ \\
\hline$g_{0}$ & $\mathfrak{S I}(p ; \boldsymbol{R})+\mathfrak{S I}(q ; \boldsymbol{R})+\boldsymbol{R}$ & $\mathfrak{S I}(p ; C)+\mathfrak{I l}(q ; \boldsymbol{C})+\boldsymbol{C}$ & $\mathfrak{S}^{*} *(2 p)+\mathfrak{S} \mathfrak{u}^{*}(2 q)+\boldsymbol{R}$ \\
\hline $\mathfrak{g}$ & & $\mathfrak{s} \mathfrak{u}(p, p)$ & \\
\hline $\mathfrak{g}_{0}$ & & $\mathfrak{B l}(p ; C)+\boldsymbol{R}$ & \\
\hline $\mathfrak{g}$ & $\mathfrak{S O}(n, n)$ & $\mathfrak{s o}(2 n ; C)$ & $\mathfrak{S D}^{*} *(4 n)$ \\
\hline$g_{0}$ & $\mathfrak{g l}(n ; \boldsymbol{R})$ & $\mathfrak{g l}(n ; C)$ & $\mathfrak{u}^{*}(2 n)$ \\
\hline $\mathfrak{g}$ & $\mathfrak{S} \mathfrak{D}(p+1, q+1)$ & $\mathfrak{s} \mathfrak{p}(p+q+2 ; C)$ & \\
\hline $\mathrm{g}_{0}$ & $\mathfrak{S} \mathfrak{v}(p+q)+\boldsymbol{R}$ & $\mathfrak{S} \mathfrak{v}(p+q ; C)+C$ & \\
\hline $\mathfrak{g}$ & $\mathfrak{B} \mathfrak{q}(n ; \boldsymbol{R})$ & $\mathfrak{s} \mathfrak{q}(n ; C)$ & $\mathfrak{s} \mathfrak{q}(n, n)$ \\
\hline$g_{0}$ & $\mathfrak{g l}(n ; \boldsymbol{R})$ & $\mathfrak{g l}(n ; C)$ & $\mathfrak{B} \mathfrak{u}(2 n)+\boldsymbol{R}$ \\
\hline
\end{tabular}

$$
\begin{array}{lll}
K=\boldsymbol{R} & K=\boldsymbol{C} & K=\boldsymbol{Q}
\end{array}
$$


The above gives the table for classical Lie algebras. The table for exceptional Lie algebras is given as following. For the detailed definition, see also [14].

$\begin{array}{llll}\mathfrak{g} & E_{6}^{1} & E_{6}^{C} & E_{6}^{4} \\ \mathfrak{g}_{0} & \mathfrak{g o}(5.5)+R & \mathfrak{g} \mathfrak{v}(10 ; C)+C & \mathfrak{s}^{\mathbf{0}}(1.9)+\boldsymbol{R} \\ \mathfrak{g} & E_{7}^{1} & E_{7}^{C} & E_{7}^{3} \\ \mathfrak{g}_{0} & E_{6}^{1}+\boldsymbol{R} & E_{6}^{C}+C & E_{6}^{4}+R\end{array}$

Proposition (7-1). Let $\mathfrak{g}=\mathfrak{g}_{-1} \oplus \mathfrak{g}_{0} \oplus \mathfrak{g}_{1}$ be a real simple graded Lie algebra such that $\mathfrak{g}$ is different from $\mathfrak{S I}(2, R)$ and $\mathfrak{I l}(2, C)$, then $H^{2,1}(\mathfrak{g})=0$.

Proof. This follows from Lemma (2-3), Lemma (3-6) and Corollary (5-3).

Proposition (7-2). Let $\mathfrak{g}=\mathfrak{g}_{-1} \oplus \mathfrak{g}_{0} \oplus \mathfrak{g}_{1}$ be a real simple graded Lie algebra such that $\mathfrak{g}$ and $\mathrm{g}_{0}$ are different from

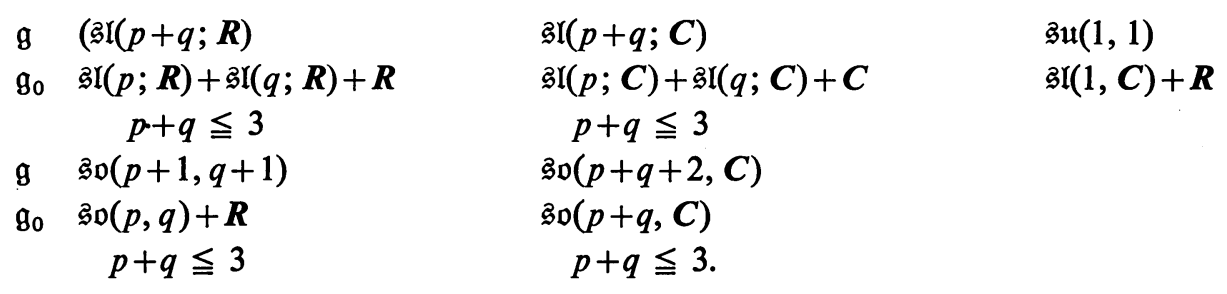

Then $H^{2,2}(g)=0$.

Proof. This follows from Lemma (2-3), Lemma (3-6) and Corollary (5-3).

Proposition (7-3). Let $\mathfrak{g}=\mathfrak{g}_{-1} \oplus \mathfrak{g}_{0} \oplus \mathrm{g}_{1}$ be a real simple graded Lie algebra. Then $H^{1,1}(\mathfrak{g})=0$ except the cases $\mathfrak{g}=\mathfrak{g} \mathfrak{I}(p+1, R), g_{0}=\mathfrak{I l}(p, R)+R$ and $\mathfrak{g}=\mathfrak{I I}(p+1, C)$, $\mathfrak{g}_{0}=\mathfrak{g l}(p, C)+C$.

Proof. This follows from Lemma (5-11) and the table above.

8. Frames of higher order contact. Let $M$ be a manifold of dimension $n$. Let $U$ and $V$ be neighborhoods of the origin 0 of $\boldsymbol{R}^{n}$. Two local diffeomorphisms $f: U \rightarrow M$ and $g: V \rightarrow M$ such that $f(0)=g(0)$ give rise to the same frame of $r$ th order contact (or $r$-frame) at $x=f(0)=g(0)$ if they have the same partial derivatives up to order $r$ at 0 . Here $1 \leqq r \leqq \infty$. The $r$-frame given by $f$ is denoted by $j^{r}(f)$. We denote by $\mathscr{F}^{r}(M)$ the set of all $r$-frames of $M$. It is a principal fibre bundle over $M$ with the natural projection $\pi ; \pi\left(j^{r}(f)\right)=f(0)$. We shall describe its structure group and the right action of the structure group on $\mathscr{F}^{r}(M)$.

Let $G^{r}(n)$ be the set of $r$-frames of $\boldsymbol{R}^{n}$ at 0 , i.e., $G^{r}(n)=\left\{j^{r}(g) \in \mathscr{F}^{r}\left(\boldsymbol{R}^{n}\right) \mid g(0)=0\right\}$. Then $G^{r}(n)$ is a group with the natural multiplication defined by $j^{r}(g) \cdot j^{r}\left(g^{\prime}\right)$ $=j^{r}\left(g \circ g^{\prime}\right)$. The group $G^{r}(n)$ acts on $\mathscr{F}^{r}(M)$ from the right by $j^{r}(f) \cdot j^{r}(g)=j^{r}(f \circ g)$ for $j^{r}(f) \in \mathscr{F}^{r}(M)$ and $j^{r}(g) \in G^{r}(n)$. It is easy to verify that $\mathscr{F}^{r}(M)$ is a principal fibre bundle over $M$ with group $G^{r}(n) . \mathscr{F}^{r}(M)$ is called the bundle of r-frame of $M$. 
Let $h: M \rightarrow N$ be a diffeomorphism from $M$ onto a manifold $N$. Then $h$ induces a bundle isomorphism $h^{(r)}: \mathscr{F}^{r}(M) \rightarrow \mathscr{F}^{r}(N)$ defined by

$$
h^{(r)}\left(j^{r}(f)\right)=j^{r}(h \circ f) \in \mathscr{F}^{r}(N) \text { for } j^{r}(f) \in \mathscr{F}^{r}(M) .
$$

We call $h^{(r)}$ the $r$ th prolongation of $h$.

From now on we shall be mainly interested in $\mathscr{F}^{2}(M)$ and $\mathscr{F}^{1}(M)$. We have a natural projection $\alpha: G^{2}(n) \rightarrow G^{1}(N)$ and $\nu: \mathscr{F}^{2}(M) \rightarrow \mathscr{F}^{1}(M)$ defined by $\alpha\left(j^{2}(g)\right)$ $=j^{1}(g)\left(j^{2}(g) \in G^{2}(n)\right)$ and $\nu\left(j^{2}(f)\right)=j^{1}(f)\left(j^{2}(f) \in \mathscr{F}^{2}(M)\right)$.

LEMMA (8-1). $\alpha: G^{2}(n) \rightarrow G^{1}(n)$ is a group homomorphism and $\nu: \mathscr{F}^{2}(M) \rightarrow$ $\mathscr{F}^{1}(M)$ is a bundle homomorphism associated with $\alpha: G^{2}(n) \rightarrow G^{1}(m)$;

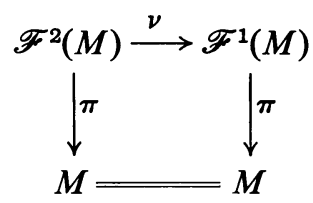

Proof. It is easy to see.

REMARK (8-1). We have a natural inclusion of $G L(n ; R)$ into $G^{r}(n)$, defined by $g \rightarrow j^{r}(g)$ for a $g \in G L(n ; R)$. In particular $G L(n ; R)$ and $G^{1}(n)$ are isomorphic by this inclusion. From now on we identify $G L(n ; \boldsymbol{R})$ with $G^{1}(n)$ and consider $G L(n ; \boldsymbol{R})$ as a subgroup of $G^{r}(n)$ by this inclusion. Then the unit element $e$ of $G L(n ; R)$ corresponds to $j^{r}(\mathrm{id}) \in G^{r}(n) \mathscr{F}^{r}\left(\boldsymbol{R}^{n}\right)$, here id means the identity transformation.

For a subgroup $G$ of $G L(n ; R)$ a $G$-subbundle $P$ of $\mathscr{F}^{1}(M)$ is called a $G$-structure on $M$. Let $P$ and $P^{\prime}$ be $G$-structures on $M$ and a manifold $M^{\prime}$ respectively. A diffeomorphism $h$ of $M$ onto $M^{\prime}$ is called an isomorphism of $P$ and $P^{\prime}$ if the first prolongation $h^{(1)}$ satisfies $h^{(1)}(P)=P^{\prime}$. Especially an isomorphism of $P$ itself is called a $G$-automorphism of $P$.

We shall now define an $\boldsymbol{R}^{n}$-values 1-form $\theta$ on $\mathscr{F}^{1}(M)$. Let $X$ be a vector tangent to $\mathscr{F}^{1}(M)$ at a point $j^{1}(f) \in \mathscr{F}^{1}(M) . f$ induces an isomorphism $f_{*}^{-1}$ of the tangent space $T(M)_{\pi(p)}$ onto $T\left(R^{n}\right)_{0}$, and $\pi_{*}\left(X_{p}\right)$ is a vector in $T(M)_{\pi(p)}$. We define $\theta$ by

$$
\left\langle X_{p}, \theta\right\rangle=f_{*}^{-1}\left(\pi_{*}\left(X_{p}\right)\right) \text {. }
$$

The restriction of $\theta$ onto a $G$-structure $P$ is also denoted by $\theta$ and called the canonical form of $P$.

Lemma (8-2). Let $P$ and $P^{\prime}$ be G-structures on $M$ and $M^{\prime}$ respectively, and $\theta$ and $\theta^{\prime}$ be their canonical forms respectively. If $h: M \rightarrow M^{\prime}$ is an isomorphism of $P$ and $P^{\prime},\left(h^{(1)}\right)^{*} \theta^{\prime}=\theta$. Conversely if a bundle isomorphism $H: P \rightarrow P^{\prime}$ satisfies $H^{*} \theta^{\prime}=\theta$, then $H$ is the first prolongation $h^{(1)}$ of the diffeomorphism $H$ of the base manifolds $M$ and $M^{\prime}$ induced by $H$.

Proof. It is easy to see (cf. [8]).

Lemma (8-3). Let $P$ be a G-structure on $M$ and $\theta$ be its canonical form. Then we have 
(8-1). $\left\langle A^{*}, \theta\right\rangle=0$ for $A \in \mathfrak{g}$,

(8-2). $R_{a}^{*} \theta=a^{-1}$ for $a \in G$.

Here $g$ denotes the Lie algebra of $G$.

Proof. It is easy to verify (cf. [8]).

Let $\mathscr{T}_{s}^{r}\left(\boldsymbol{R}^{n}\right)$ be the vector space of all tensors of type $(r, s)$ on $\boldsymbol{R}^{n}$, i.e., $\mathscr{T}_{s}^{r}\left(\boldsymbol{R}^{n}\right)$ $=\left(\otimes^{r} \boldsymbol{R}^{n}\right) \otimes\left(\bigotimes^{s}\left(\boldsymbol{R}^{n}\right)^{*}\right)$. The group $G L(n ; \boldsymbol{R})$ naturally operates on $\mathscr{T}_{s}^{r}\left(\boldsymbol{R}^{n}\right)$. For a $G$-structure $P$ on $M$, a tensor field of type $(r, s)$ on $M$ is a mapping $\mathfrak{x}$ of $P$ into $T_{s}^{r}\left(R^{n}\right)$ satisfying $\mathfrak{x}_{p a}=a^{-1}$. ( $\left.\mathfrak{x}_{p}\right)$ for all $a \in G$ and $p \in P$. This definition of a tensor field on $M$ is equivalent to the usual one.

Let $H$ be a subgroup of $G^{2}(n)$. An $H$-subbundle $Q$ of $\mathscr{F}^{2}(M)$ is called an $H$ structure of 2 nd order on $M$. Then $\nu(Q) \subset \mathscr{F}^{1}(M)$ is an $\alpha(H)$-structure on $M$, which is called the associated $\alpha(H)$-structure of $Q$. Let $Q$ and $Q^{\prime}$ be $H$-structure of 2nd order on $M$ and $M^{\prime}$ respectively. A diffeomorphism $h: M \rightarrow M^{\prime}$ is called an isomorphism of $Q$ and $Q^{\prime}$ if $h^{(2)}(Q)=Q^{\prime}$. Especially an isomorphism of $Q$ itself is called an $H$-automorphism of $Q$.

We shall now define a 1 -form $\Theta$ on $\mathscr{F}^{2}(M)$ with values in $T\left(\mathscr{F}^{1}\left(\boldsymbol{R}^{n}\right)\right)_{e}$. Let $X$ be a vector tangent to $\mathscr{F}^{2}(M)$ at $j^{2}(f)$. Since $f$ is a diffeomorphism of a neighborhood of the origin $0 \in \boldsymbol{R}^{n}$ onto a neighborhood of $f(0) \in M$, it induces a diffeomorphism $f^{(1)}$ of a neighborhood of $e \in \mathscr{F}^{1}\left(\boldsymbol{R}^{n}\right)$ onto a neighborhood of $j^{1}(f) \in$ $\mathscr{F}^{1}(M) . f^{(1)}$ then induces an isomorphism $f_{*}^{(1)}$ of the tangent space $T\left(\mathscr{F}^{1}\left(\boldsymbol{R}^{n}\right)\right)_{e}$ at $e$ onto the tangent space of $\mathscr{F}^{1}(M)$ at $\nu\left(j^{2}(f)\right)=j^{1}(f)$. Then form $\Theta$ is defined by

$$
\langle X, \Theta\rangle=\left(f_{*}^{(1)}\right)^{-1}\left(\nu_{*}(X)\right),
$$

and is called the canonical form of $\mathscr{F}^{2}(M)$. For an $H$-structure of 2 nd order $Q$, the restriction of $\Theta$ onto $Q$ is also denoted by $\Theta$ and called the canonical form of $Q$.

LEMMA (8-4). Let $\Theta$ and $\Theta^{\prime}$ be the canonical forms of $\mathscr{F}^{2}(M)$ and $\mathscr{F}^{2}\left(M^{\prime}\right)$ respectively. Let $h: M \rightarrow M^{\prime}$ be a diffeomorphism. Then the second prolongation $h^{(2)}$ of $h$ satisfies

$$
\left(h^{(2)}\right) * \Theta^{\prime}=\Theta .
$$

Conversely if $H: \mathscr{F}^{2}(M) \rightarrow \mathscr{F}^{2}\left(M^{\prime}\right)$ is a bundle isomorphism such that $H^{*} \Theta^{\prime}=\Theta$, then $H$ is the second prolongation $h^{(2)}$ of the diffeomorphism $H$ of the base manifolds $M$ and $M^{\prime}$ induced by $H$.

Proof. See [8].

We define an action of $G^{2}(n)$ on $T\left(\mathscr{F}^{1}\left(\boldsymbol{R}^{n}\right)\right)_{e}$. Let $j^{2}(g) \in G^{2}(n)$ and $j^{1}(f) \in \mathscr{F}^{1}\left(\boldsymbol{R}^{n}\right)$. The mapping of a neighborhood of $e$ onto a neighborhood of $e$ defined by

$$
j^{1}(f) \mapsto j^{1}\left(g \circ f \circ g^{-1}\right)
$$

induces a linear isomorphism of the tangent space $T\left(\mathscr{F}^{1}\left(R^{n}\right)\right)_{e}$ onto itself. This linear isomorphism depends only on $j^{2}(g)$ and is denoted by $\operatorname{Ad}\left(j^{2}(g)\right)$. 
LeMma (8-5). Let $\Theta$ be the canonical form on $\mathscr{F}^{2}(M)$. Then

(8-3). $\left\langle A^{*}, \Theta\right\rangle=\alpha_{*}(A)$ for $A \in \mathfrak{g}^{2}(n)$,

(8-4). $\left(R_{a}^{*}\right) \Theta=\operatorname{Ad}\left(a^{-1}\right) \cdot \Theta$ for $a \in G^{2}(n)$.

Here $\mathfrak{g}^{2}(n)$ denotes the Lie algebra of $G^{2}(n)$ and $\alpha_{*}$ is the linear mapping: $\mathfrak{g}^{2}(n) \rightarrow$ $\mathfrak{g l}(n, R)$ induced by $\alpha: G^{2}(n) \rightarrow G L(n ; R)$.

Proof. See [8].

Lemma (8-6). For an $A \in \mathfrak{g}^{2}(n)$ we have $\nu_{*}\left(A^{*}\right)=\left(\alpha_{*} A\right)^{*}$.

Proof. The proof is straightforward.

LEMMA (8-7). Let $\Theta$ and $\theta$ be the canonical forms of $\mathscr{F}^{2}(M)$ and $\mathscr{F}^{1}(M)$ respectively. Then we have

$$
\nu_{*}^{\prime}(\Theta)=\nu^{*} \theta .
$$

Here $\nu: \mathscr{F}^{2}(M) \rightarrow \mathscr{F}^{1}(M)$ and $\nu^{\prime}: \mathscr{F}^{2}\left(\boldsymbol{R}^{n}\right) \rightarrow \mathscr{F}^{1}\left(\boldsymbol{R}^{n}\right)$.

Proof. Let $X$ be a vector tangent to $\mathscr{F}^{2}(M)$ at $j^{2}(f)$. Then

$$
\left\langle X, \nu_{*}^{\prime}(\Theta)\right\rangle=\nu_{*}^{\prime}\langle X, \Theta\rangle=\nu_{*}^{\prime}\left(\left(f_{*}^{(1)}\right)^{-1}\left(\nu_{*} X\right)\right)=f_{*}^{-1}\left(\pi_{*}\left(\nu_{*} X\right)\right)=\left\langle X, \nu^{*} \theta\right\rangle .
$$

Let $A(n ; \boldsymbol{R})$ be the affine group acting on $\boldsymbol{R}^{n}$. Considering $A(n ; \boldsymbol{R})$ as a principal bundle over $\boldsymbol{R}^{n}=A(n ; \boldsymbol{R}) / G L(n ; \boldsymbol{R})$ with structure group $G L(n ; \boldsymbol{R})$, we have a natural bundle isomorphism between $A(n ; \boldsymbol{R})$ and $\mathscr{F}^{1}\left(\boldsymbol{R}^{n}\right)$ :

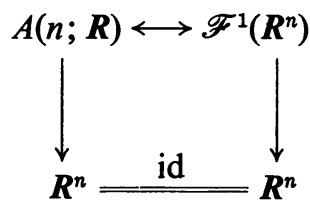

Under this isomorphism the identity $e$ of $A(n ; R)$ (or of $G L(n ; R)$ ) corresponds to $j_{0}^{1}(\mathrm{id})$. The tangent space of $\mathscr{F}^{1}\left(\boldsymbol{R}^{n}\right)$ at $e$, corresponds to the Lie algebra

$$
\mathfrak{a}(n ; \boldsymbol{R})=\boldsymbol{R}^{n}+\mathfrak{g l}(n ; \boldsymbol{R}) \quad \text { of } A(n ; \boldsymbol{R}) .
$$

Let $\Theta$ be the canonical form of $\mathscr{F}^{2}(M)$. We denote by $\Theta_{-1}$ and $\Theta_{0}$ the $\boldsymbol{R}^{n}$ - and $\mathfrak{g l}(n ; \boldsymbol{R})$-component of $\Theta$.

Lemma (8-8). We have $d \Theta_{-1}+\left[\Theta_{-1}, \Theta_{0}\right]=0$.

Proof. See [12].

9. Flat $L_{0}$-structures of 2 nd order. For a linear subgroup $G$ of $G L(n, R)$, the subgroup $\boldsymbol{R}^{n} \cdot G \subset A(n, R)$ can be considered a $G$-structure on $\boldsymbol{R}^{n}$. This structure is called a flat $G$-structure. A $G$-structure $P$ over $M$ is called flat if it is locally equivalent to the flat $G$-structure.

Let $L / L_{0}$ be a semisimple homogeneous space, and $\mathfrak{l}=\mathfrak{g}_{-1} \oplus \mathfrak{g}_{0} \oplus \mathfrak{g}_{1}$ be its associated graded Lie algebra. We denote by $\bar{o}$ the point of $\left\{L_{0}\right\}$. Then the mapping Exp from $\mathfrak{g}_{-1}$ into $L / L_{0}$ defined by $\operatorname{Exp}(x)=\exp x \cdot \bar{o}$ is a diffeomorphism into. 
We have a natural mapping ` from $L$ into $\mathscr{F}^{2}\left(L / L_{0}\right)$ and a group homomorphism $\iota^{\prime}: L / L_{0} \rightarrow G^{2}(n)$ by

$$
\begin{aligned}
& l \in L \mapsto \iota(l)=j^{2}(l \cdot \operatorname{Exp}), \\
& a \in L_{0} \mapsto \iota^{\prime}(a)=j^{2}\left(\operatorname{Exp}^{-1} \cdot a \cdot \operatorname{Exp}\right) .
\end{aligned}
$$

Lemma (9-1). $\iota^{\prime}$ is injective. In particular $\iota$ is injective.

Proof. Let $N$ be the kernel of $\iota^{\prime}$, and $\mathfrak{n}$ be the Lie algebra of $N$. Since $N$ is contained in the kernel of the linear isotropy representation, we get $N \subset G_{1}, \mathfrak{n} \subset \mathfrak{g}_{1}$ (Lemma (6-5)). And it is clear $\mathfrak{n}=\left\{x \in \mathfrak{g}_{1} \mid[\mathfrak{g}, x] \subset \mathfrak{g}_{1}\right\}$. Then by the same argument in Proposition (3-1), we have $\mathfrak{n}=0$. Thus $N$ is a discrete group. In view of Lemma (6-1), we put $N=\exp \mathrm{m}$. It is clear that $G_{0}$ normalizes $N$. Therefore, $N$ being discrete, we have $\left[g_{0}, \mathfrak{m}\right]=0$. Hence $\mathfrak{m}=0$ by Lemma (3-4).

Thus $L \rightarrow L / L_{0}$ can be naturally considered as an $L_{0}$-structure of 2 nd order (if we fix $\left.\mathfrak{g}_{-1}\right)$. We call this structure the flat $L_{0}$-structure of 2 nd order. An $L_{0}$-structure of 2 nd order is called flat if it is locally equivalent of the flat $L_{0}$-structure.

We recall that $G^{2}(n)$ operates on $\mathfrak{g}_{-1} \oplus \mathfrak{g l}\left(\mathfrak{g}_{-1}\right)$, identifying $\mathfrak{g}_{-1}$ with $\boldsymbol{R}^{n}(n=$ $\left.\operatorname{dim} \mathfrak{g}_{-1}\right)$. With this in mind, we have the following.

Lemma (9-2). The group $L_{0}\left(\subset G^{2}(n)\right)$ leaves $\mathrm{g}_{-1} \oplus \mathrm{g}_{0}\left(\subset \mathrm{g}_{-1} \oplus \mathrm{gl}\left(\mathrm{g}_{-1}\right)\right)$ invariant And its operation onto $\mathfrak{g}_{-1} \oplus \mathfrak{g}_{0}$ is identical to the operation of $L_{0}: \mathfrak{l} / \mathfrak{g}_{-1} \rightarrow \mathfrak{l} / \mathfrak{g}_{-1}$ induced by $\operatorname{Ad}(a): \mathfrak{l} \rightarrow \mathfrak{l}$.

Proof. This is trivial.

Let $\Xi$ be the Mauer-Cartan form of the group $L$. We write $\Xi$ as $\Xi=\Xi_{-1} \oplus \Xi_{0}$ $\oplus \Xi_{1}$ according to the decomposition $\mathfrak{l}=\mathfrak{g}_{-1} \oplus \mathfrak{g}_{0} \oplus \mathfrak{g}_{1}$.

LEMMA (9-3). Let $\Theta=\Theta_{-1} \oplus \Theta_{0}$ be the canonical form of the flat $L_{0}$-structure of 2 nd order $L \rightarrow L / L_{0}$. Then we have $\Theta_{-1}=\Xi_{-1}$ and $\Theta_{0}=\Xi_{0}$.

Proof. This follows from the definition of the Mauer-Cartan form.

Let $L \rightarrow L / L_{0}$ be the flat $L_{0}$-structure of 2 nd order. Then $v(L) \subset \mathscr{F}^{1}\left(L / L_{0}\right)$ is a $G_{0}$-structure, here $G_{0}$ is the linear isotropy subgroup and $\nu: \mathscr{F}^{2}\left(L / L_{0}\right) \rightarrow \mathscr{F}^{1}\left(L / L_{0}\right)$.

Lemma (9-4). The $G_{0}$-structure $\nu(L) \rightarrow L / L_{0}$ is flat.

Proof. This follows from Lemma (6-5).

10. Linear connections and 2 nd order structures. An affine connection $\Gamma$ on a manifold $M$ of dimension $n$ is a $\mathfrak{g l}(n ; R)$-values 1 -form on $\mathscr{F}^{1}(M)$ satisfying the conditions

(10-1). $\left\langle A^{*}, \Gamma\right\rangle=A$ for $A \in \operatorname{gl}(n ; R)$,

(10-2). $R_{c}^{*} \Gamma=\operatorname{Ad}\left(a^{-1}\right) \Gamma$ for $a \in G L(n ; R)$.

For an element $u \in \boldsymbol{R}^{n}$, there exists uniquely a vector field $V_{u}$ on $\mathscr{F}^{1}(N)$ such that $\left\langle V_{u}, \Gamma\right\rangle=0$ and $\left\langle V_{u}, \theta\right\rangle=u$, where $\theta$ is the canonical form of $\mathscr{F}^{1}(M)$. We call this 
vector field $V_{u}$ the horizontal vector field of $u \in \boldsymbol{R}^{n}$. The torsion form $T(\Gamma)$ and the curvature form $R(\Gamma)$ are defined by

(10-3). $d \theta=[\theta, \Gamma]+T(\Gamma)$,

(10-4). $d \Gamma=\frac{1}{2}[\Gamma, \Gamma]+R(\Gamma)$.

If $T(\Gamma)=0, \Gamma$ is called an affine connection with torsion. We define the curvature tensor field $\mathfrak{x}$ of type (1.3) by

$$
\mathfrak{r}_{p}(u, v)=\left\langle V_{u} \wedge V_{v}, R(\Gamma)\right\rangle \text { for } u, v \in R^{n} .
$$

We define the covariant derivative of a tensor field $\mathfrak{x}$ of type $(r, s)$ on $M$. At each point $p \in \mathscr{F}^{1}(M)$, let $\nabla$ be the linear mapping of $\boldsymbol{R}^{n}$ into $\mathscr{T}_{s}^{r}\left(\boldsymbol{R}^{n}\right)$ defined by

$$
\nabla_{u} \mathfrak{x}=\left(V_{u}\right)_{p} \cdot \mathfrak{x} .
$$

Then $\nabla \mathfrak{x}$ is a tensory field of type $(r, s+1)$.

A cross-section $s: \mathscr{F}^{1}(M) \rightarrow \mathscr{F}^{2}(M)$ is called admissible if we have (10-5). $s(p \cdot a)=s(p) \cdot a$ for $p \in \mathscr{F}^{1}(M)$ and $a \in G L(n ; R)$.

TheOREM (10-1) (S. KobayASHI [12]). For an admissible cross-section $s: \mathscr{F}^{1}(M)$ $\rightarrow \mathscr{F}^{2}(M) . s^{*} \Theta_{0}$ is an affine connection on $M$ without torsion. And this gives us one-to-one correspondence between affine connections on $M$ without torsion and admissible cross-sections.

Let $P$ be a $G$-structure on $M$. An affine connection $\Gamma$ on $M$ is called a $G$-connection if the restriction of $\Gamma$ onto $P$ has values in $g$ (the Lie algebra of $G$ ).

Let $L / L_{0}$ be a semisimple flat homogeneous space. We fix once and for all its associated graded Lie algebra $\mathfrak{l}=\mathfrak{g}_{-1} \oplus \mathfrak{g}_{0} \oplus \mathfrak{g}_{1}$. Then $L_{0}$ is a subgroup of $G^{2}(n)$. We denote by $G_{0}$ the linear isotropy subgroup of $L / L_{0} ; G_{0} \subset L_{0} \subset G^{2}(n)$.

Let $P$ be a $G_{0}$-structure on $M$ and $\Gamma$ be a $G_{0}$-connection on $P$ without torsion. By Theorem (10-1), $P$ is a $G_{0}$-subbundle of $\mathscr{F}^{2}(M)$. By extending the structure group $G_{0}$ into $L_{0}$, we have a $L_{0}$-structure of 2 nd order, which is called the $L_{0^{-}}$ structure of 2 nd order associated with a $G_{0}$-connection $\Gamma$, and denoted by $Q(\{\Gamma\})$.

Two $G_{0}$-connections $\Gamma$ and $\Gamma^{\prime}$ on $P$ without torsion are called $L_{0}$-equivalent if $Q(\Gamma)=Q\left(\Gamma^{\prime}\right)$. This is clearly equivalence relations. Denote by $A(P)$ the set of all $G_{0}$-connection on $P$ without torsion, each equivalence class $\mathfrak{a}$ in $A(P)$ define uniquely a $L_{0}$-structure of 2 nd order, which is denoted by $Q(\mathfrak{a})$.

LEMMA (10-1). Let $L / L_{0}$ be a semisimple flat homogeneous space with the associated graded Lie algebra $\mathfrak{l}=\mathfrak{g}_{-1} \oplus \mathfrak{g}_{0} \oplus \mathfrak{g}_{1}$, and $G_{0}$ be its linear isotropy group. Let $P \rightarrow M$ be a $G_{0}$-structure and $\mathfrak{a}$ an $L_{0}$-equivalence class in $A(P)$. If we denote by $\Theta=\Theta_{-1} \oplus \Theta_{0}$ the canonical form of $Q(\pi)$, the associated $L_{0}$-structure of $\pi$, then we have

(1) $\Theta_{-1}$ is $\mathrm{g}_{-1}$-valued and $\Theta_{0}$ is $\mathrm{g}_{0}$-valued,

(2) $\left\langle A^{*}, \Theta_{0}\right\rangle=A^{\prime}$ (the g-component of $A$ ), for $A \in \mathfrak{g}_{0} \oplus \mathfrak{g}_{1}$,

(3) $R_{a}^{*}\left(\Theta_{-1} \oplus \Theta_{0}\right) \equiv \operatorname{Ad}\left(a^{-1}\right)\left(\Theta_{-1}+\Theta_{0}\right) \bmod g_{1}$. 
Proof. (1) Let $s: P \rightarrow Q(\mathfrak{a})$ be the imbedding which gives one of $G_{0}$-connection in $a$. Then the restriction of $\Theta_{-1}$ and $\Theta_{0}$ have their values in $g_{-1}$ and $g_{0}$ respectively. Our assertion follows from Lemma (9-2). The rest of our assertion follows also from Lemma (9-2) and the definitions.

Let $f: M \rightarrow M$ be a $G_{0}$-automorphism of a $G_{0}$-structure $P$. Then for each element $\Gamma$ in $A(P), f_{*}^{(1)} \Gamma$ is also a $G_{0}$-connection on $P$. For each equivalence class $\mathfrak{a}$ of $A(P), f$ is called $\mathfrak{a}$-automorphism if $f_{*}^{(1)} \mathfrak{a}=\mathfrak{a}$.

Proposition (10-1). If $H^{1,1}(\mathfrak{l})$ is zero, then there is only one equivalence class in $A(P)$ if $A(P) \neq \varnothing$.

Proof. Let $\Gamma$ and $\Gamma^{\prime}$ be two connections in $A(P)$. Since $\Gamma-\Gamma^{\prime}$ is zero on the fibres of $P \rightarrow M$, at each point $p$ of $P$, there exists a function $F_{p}$ from $\boldsymbol{R}^{n}$ into $\mathrm{g}_{0}$ such that $\left\langle X, \Gamma-\Gamma^{\prime}\right\rangle=F_{p}(\langle X, \theta\rangle)$ for each vector. We consider $F_{p}$ as an element of $C^{1,1}(\mathfrak{l})$. Since $\Gamma$ and $\Gamma^{\prime}$ are torsion free, we have $\partial F_{p}=0$ for $p \in P$. Since $H^{1,1}(\mathfrak{l})=0$ there exists $F_{p}^{\prime} \in C^{2,0}(\mathfrak{l})$ such that $\partial F_{p}^{\prime}=F_{p}$. If $s$ and $\bar{s}$ are admissible cross-sections which give $\Gamma$ and $\Gamma^{\prime}$ respectively, then it is easy to see that $s^{\prime}(p)=s(p) \exp F_{p}^{\prime}$.

REMARK. For the geometric meaning of his proposition, see 7.

11. Normal Cartan connections. Let $L / L_{0}$ be a connected homogeneous space on which a Lie group $L$ operates effectively and transitively. We denote by $\mathfrak{l}$ and $\mathfrak{I}_{0}$ the Lie algebra of $L$ and $L_{0}$. A Cartan connection of type $L / L_{0}$ on an $L_{0}$-principal bundle $Q \rightarrow M$ is, by definition, an I-valued 1-form $\omega$ on $Q$ satisfying the conditions;

(11-0). $\operatorname{dim} M=\operatorname{dim} L / L_{0}$,

(11-1). $\left\langle A^{*}, \omega\right\rangle=A$ for $A \in \mathfrak{I}_{0}$,

(11-2). $R_{a}^{*} \omega=\operatorname{Ad}\left(a^{-1}\right) \omega$ for $a \in L_{0}$,

(11-3). $\langle X, \omega\rangle \neq 0$ for every nonzero vector of $Q$.

Let $H: Q \rightarrow Q$ be a bundle isomorphism. We call $H$ an isomorphism of the Cartan connection $\omega$ if we have $H^{*} \omega=\omega$. We define the curvature form $\Omega=\Omega(\omega)$ of $\omega$ by $\Omega=d \omega+\frac{1}{2}[\omega, \omega]$.

LEMMA (11-1). We have

(1) $R_{a}^{*} \Omega=\operatorname{Ad}\left(a^{-1}\right) \Omega$,

(2) $i\left(A^{*}\right) \Omega=0$ for $A \in \mathfrak{I}_{0}$, here $i\left(A^{*}\right) \Omega$ means the inner product of $\Omega$ and $A^{*}$.

Proof. This is well known [15].

EXAmple (11-1). Let $\omega_{0}$ be the Mauer-Cartan form of $L$. Then $\omega_{0}$ is a Cartan connection on the $L_{0}$-principal bundle $L \rightarrow L / L_{0}$.

Proposition (11-1). Let $\omega$ be a Cartan connection on an $L_{0}$-principal bundle $Q \rightarrow M$. If the curvature form $\Omega$ of $\omega$ is zero, then at each point $m \in M$, there exists a local bundle isomorphism $H$ between $Q$ and $L$, defined on some neighborhood of m such that $H^{*} \omega_{0}=\omega$.

Proof. Since $\Omega$ is zero, the 1 -form $\omega$ satisfies $d \omega+\frac{1}{2}[\omega, \omega]=0$. It follows that, given a point $u$ of $Q$, there exists a local diffeomorphism $H$ of a neighborhood $N^{\prime}$ 
of the identity of $L$ onto a neighborhood $N$ of $u$ which sends $\omega$ into the MauerCartan form $\omega_{0}$. Then it is easy to see that in an obvious manner, we extend $H$ to a diffeomorphism $H: N^{\prime} \cdot L_{0} \rightarrow H \cdot L_{0}$. Let $U^{\prime}=\pi^{\prime}\left(N^{\prime}\right)$ and $U=\pi(N)$, where $\pi^{\prime}$ : $L \rightarrow L / L_{0}$ and $\pi: Q \rightarrow M$. Then $\pi^{\prime-1}\left(U^{\prime}\right)=N^{\prime} \cdot L_{0}$, and $\pi^{-1}(U)=N \cdot L_{0}$. By construction $H: \pi^{-1}(U) \rightarrow \pi^{-1}(U)$ is a bundle isomorphism.

From now on we assume furthermore that $L / L_{0}$ is a semisimple flat homogeneous space. We denote by $\mathfrak{l}=\mathfrak{g}_{-1} \oplus \mathfrak{g}_{0} \oplus \mathfrak{g}_{1}$ its associated graded Lie algebras and by $G_{0}$ its linear isotropy subgroup of $L_{0}$. Then a Cartan connection $\omega$ and its curvature form $\Omega$ can be written respectively as

$$
\omega=\omega_{-1} \oplus \omega_{0} \oplus \omega_{1} \text { and } \Omega=\Omega_{-1} \oplus \Omega_{0} \oplus \Omega_{1}
$$

according to the decomposition; $\mathfrak{l}=\mathfrak{g}_{-1} \oplus \mathfrak{g}_{0} \oplus \mathfrak{g}_{1}$. Then clearly we have the following relations.

(11-4). $\Omega_{-1}=d \omega_{-1}+\left[\omega_{0}, \omega_{-1}\right]$.

(11-5). $\Omega_{0}=d \Omega_{0}+\frac{1}{2}\left[\omega_{-1}, \omega_{1}\right]$.

(11-6). $\Omega_{1}=d \omega_{1}+\left[\omega_{1}, \omega_{0}\right]$.

Lemma (11-2). If $\Omega_{-1}=0$, for an element $a \cdot \exp z \in L_{0}\left(a \in G_{0}, z \in \mathfrak{g}_{-1}\right)$, we have (11-7). $\left(R_{a} \cdot \exp z\right)^{*} \Omega_{0}=\operatorname{Ad}\left(a^{-1}\right) \Omega_{0}$,

$(11-8) .\left(R_{a} \cdot \exp z\right)^{*} \Omega_{1}=-\left[z, \Omega_{0}\right]+\operatorname{Ad}\left(a^{-1}\right) \Omega_{1}$.

Proof. By Lemma (11-1), we have

$$
\begin{aligned}
\left(R_{a} \exp z\right)^{*}\left(\Omega_{0} \oplus \Omega_{1}\right) & =\operatorname{Ad}\left(\exp (-z) \cdot a^{-1}\right)\left(\Omega_{0} \oplus \Omega_{1}\right) \\
& =\operatorname{Ad}(\exp (-z)) \cdot \operatorname{Ad}\left(a^{-1}\right)\left(\Omega_{0} \oplus \Omega_{1}\right) \\
& =\exp \operatorname{ad}(-z)\left(\operatorname{Ad}\left(a^{-1}\right) \Omega_{0}+\operatorname{Ad}\left(a^{-1}\right) \Omega_{1}\right) \\
& =\operatorname{Ad}\left(a^{-1}\right) \Omega_{0}+\operatorname{Ad}\left(a^{-1}\right) \Omega_{1}-\left[z, \Omega_{0}\right] .
\end{aligned}
$$

Lemma (11-3). Let $P \rightarrow M$ be an $G_{0}$-structure on $M$, and $\mathfrak{a}$ be a $L_{0}$-equivalence class in $A(P)$. Then there exists at least one Cartan connection $\omega=\omega_{-1} \oplus \omega_{0} \oplus \omega_{1}$, of type $L / L_{0}$ on the associated $L_{0}$-structure $Q(\mathfrak{a})$ of 2 nd order such that $\omega_{-1}=\Theta_{-1}$ and $\omega_{0}=\Theta_{0}$, here $\Theta=\Theta_{-1} \oplus \Theta_{0}$ is the canonical forms of $Q(\mathfrak{a})$.

Proof. This is a local problem. In fact let $\left\{U_{\lambda}\right\}$ be a locally finite open covering of $M$ with a partition of unity $\left\{f_{\lambda}\right\}$. If $\omega_{\lambda}$ is a Cartan connection on $Q(\mathfrak{a}) U_{\lambda}$ with the given $\Theta_{-1}$ and $\Theta_{0}$, then $\sum_{\lambda}\left(f_{\lambda} \circ \pi\right) \omega_{\lambda}$ is a Cartan connection on $Q(\mathfrak{a})$ satisfying our condition, where $\pi: Q(\mathfrak{a}) \rightarrow M$ is a projection. Hence our problem is reduced to the case where $Q(\mathfrak{a})$ is a trivial bundle. Fix a cross-section $s: P \rightarrow Q$ such that $s(p a)=s(p) a$ for $p \in P$ and $a \in G_{0}$, and set $\left\langle X, \omega_{1}\right\rangle=0$ for every vector tangent to $s(P)$. If $Y$ is an arbitrary vector tangent to $Q(\mathfrak{a})$, then we can write uniquely

(11-9). $U=R_{a}(X)+V$

where $X$ is a vector tangent to $s(P)$ and $a \in \exp g_{1}$. And finally $V$ is a vector tangent to a fibre of $Q \rightarrow P$ so that $V$ can be extended to a unique fundamental vector field $A^{*}$ on $Q$ with $A \in \mathfrak{g}_{1}$. Then we put 


$$
\left\langle Y, \omega_{1}\right\rangle=g_{1} \text {-component of } \operatorname{Ad}\left(a^{-1}\right)\left\langle X, \Theta_{-1} \oplus \Theta_{0}\right\rangle+A .
$$

Then $\omega=\Theta_{-1} \oplus \Theta_{0} \oplus \omega_{1}$ is a Cartan connection. In fact for $A \in \mathfrak{g}_{0}$ and $B \in \mathfrak{g}_{1}$

$$
\left\langle A^{*}+B^{*}, \omega\right\rangle=A+\left\langle A^{*}+B^{*}, \omega_{1}\right\rangle=A+B \quad(\operatorname{Lemma}(8-6)) \text {. }
$$

For $\exp z\left(z \in g_{1}\right)$ and $Y$ in (11-9),

$$
\begin{aligned}
\left\langle Y,(R \exp z)^{*} \omega\right\rangle= & \left\langle(R \exp z)_{*} Y, \omega\right\rangle=\left\langle\left(R_{a} \exp z\right)_{*} X+A^{*}, \omega\right\rangle \\
= & \left\langle\left(R_{1} \exp z\right)_{*} X+A, \Theta_{-1} \oplus \Theta_{0}\right\rangle \\
& + \text { the } g_{1} \text {-component of } \operatorname{Ad}\left(\exp (-z) a^{-1}\right)\left\langle X, \Theta_{-1} \oplus \Theta_{0}\right\rangle+A \\
= & \operatorname{Ad}\left(\exp (-z) a^{-1}\right)\left\langle X, \Theta_{-1}+\Theta_{0}\right\rangle+A \quad(\text { from Lemma }(9-2)) \\
= & \operatorname{Ad}(\exp (-z))\langle Y, \omega\rangle .
\end{aligned}
$$

Hence $\left(R_{\exp z}\right) * \omega=\operatorname{Ad}(\exp (-z)) \omega$. For a $g \in G_{0}$,

$$
\begin{aligned}
\left\langle Y, R_{g}^{*} \omega\right\rangle & =\left\langle R_{g} Y, \omega\right\rangle=\left\langle R_{g^{*}} R_{a^{*}} X+R_{g^{*}} A, \omega\right\rangle \\
& =\left\langle R_{g^{*}} R_{a^{*}} R_{g^{*}-1} R_{g^{*}} X+\left(\operatorname{Ad}\left(g^{-1}\right) A\right)^{*}, \omega\right\rangle \\
& =\operatorname{Ad}\left(g^{-1} a g\right)^{-1}\left\langle R_{g^{*}} X, \omega\right\rangle+\operatorname{Ad}\left(g^{-1}\right) A \quad\left(g^{-1} a g \in \exp g_{1}\right) \\
& =\operatorname{Ad}\left(g^{-1} a g\right)^{-1}\left\langle R_{g^{*}} X, \Theta_{-1} \oplus \Theta_{0}\right\rangle+A d\left(g^{-1}\right) A
\end{aligned}
$$

(since $R_{g^{*}} X$ is tangent to $S(P)$ )

$$
\begin{aligned}
& =\operatorname{Ad}\left(g^{-1} a g\right)^{-1} \operatorname{Ad}\left(g^{-1}\right)\left\langle X, \Theta_{-1} \oplus \Theta_{0}\right\rangle+\operatorname{Ad}\left(g^{-1}\right) A \\
& =\operatorname{Ad}\left(g^{-1} a^{-1}\right)\left\langle X, \Theta_{-1} \oplus \Theta_{0}\right\rangle+\operatorname{Ad}\left(g^{-1}\right) A \\
& =\operatorname{Ad}\left(g^{-1}\right)\left\{\operatorname{Ad}\left(a^{-1}\right)\left\langle K, \Theta_{-1} \oplus \Theta_{0}\right\rangle+A\right\} \\
& =\operatorname{Ad}\left(g^{-1}\right)\langle Y, \omega\rangle .
\end{aligned}
$$

Thus we have shown that the conditions (11-1) and (11-2) are satisfied. Now since $\langle Y, \omega\rangle=\operatorname{Ad}\left(a^{-1}\right)\left\langle X, \Theta_{-1} \oplus \Theta_{0}\right\rangle+A,\langle Y, \omega\rangle=0$ implies $Y=0$, proving the condition (11-3).

A Cartan connection which satisfies the assumption of Lemma (11-3) is called an admissible Cartan connection on $Q(\mathfrak{a})$.

- Let now $\omega=\Theta_{-1} \oplus \Theta_{0} \oplus \omega_{1}$ be an admissible Cartan connection on $Q(\mathfrak{a})$, and $\Omega=\Omega_{-1} \oplus \Omega_{0} \oplus \Omega_{1}$ be its curvature form.

Lemma (11-4). We have $\Omega_{-1}=0$. And there exists a form $\Omega$ on $P$ such that $\nu^{*} \widetilde{\Omega}=\Omega_{0}$.

Proof. By (11-4), $\Omega_{-1}=d \Theta_{-1}+\left[\Theta_{0}, \Theta_{-1}\right]$. Then by Lemma (8-8) we have $\Omega_{-1}=0$. The rest of our assertion follows from (11-1).

At each point $q \in Q$, we define a subspace $H_{q}$ of $T(Q)_{q}$ by

$$
H_{q}=\left\{X \in T(Q)_{q} \mid\left\langle X, \Theta_{0}\right\rangle=0,\left\langle X, \omega_{1}\right\rangle=0\right\} .
$$

Lemma (11-5). We have $T(Q)_{q}=H_{q} \oplus\left(\mathrm{g}_{0} \oplus \mathrm{g}_{1}\right)^{*}$ (direct sum), and $\Theta_{-1}$ is a linear isomorphism from $\mathrm{H}_{q}$ onto $\mathfrak{g}_{-1}$. 
Proof. From the condition (11-3), $H_{q} \cap\left(\mathfrak{g}_{0} \oplus \mathfrak{g}_{1}\right)^{*}=\{0\}$. Take any element $X \in T(Q)_{q}$. Then $X-\left(\left\langle X, \Theta_{0}+\omega_{1}\right\rangle\right)^{*}$ belongs to $H_{q}$. The surjectivity of $\Theta_{-1}$ follows from Lemma (8-7), and the injectivity follows from the condition (11-3).

Now we define mappings $c=c(\omega)$ and $v=b(\omega)$ from $Q$ into $C^{1,2}(\mathfrak{l})$ and $C^{2,2}(\mathfrak{l})$ respectively by

$$
c_{q}(u, v)=\left\langle X \wedge Y, \Omega_{0}\right\rangle \text { and } b_{q}(u, v)=\left\langle X \wedge Y, \Omega_{1}\right\rangle,
$$

where $X, Y \in H_{q}$ are defined by $\left\langle X, \Theta_{-1}\right\rangle=u$ and $\left\langle Y, \Theta_{-1}\right\rangle=v$.

LEMMA (11-6). We have

(1) $c_{q \cdot a \exp z}=\rho(a)^{-1} c_{q}$ for $a \in G_{0}, z \in \mathfrak{g}_{1}$,

(2) $\partial c_{q}=0$ for $q \in Q(\mathfrak{a})$,

(3) if $\Omega_{0}=0$, then $\partial b_{q}=0$ for $q \in Q(\mathfrak{a})$.

Proof. First we remark that $c_{q}(u, v)=\left\langle X^{\prime} \wedge Y^{\prime}, \Omega_{0}\right\rangle$ if $\left\langle X^{\prime}, \Theta_{-1}\right\rangle=u$ and $\left\langle Y^{\prime}, \Theta_{-1}\right\rangle=v$. In fact let $X \in H_{q}$ and $Y \in H_{q}$ be such that $\left\langle X, \Theta_{-1}\right\rangle=u$ and $\left\langle Y, \Theta_{-1}\right\rangle=v$. Then $X-X^{\prime}=A^{*}$ and $Y-Y^{*}=B^{*}$ for $A, B \in g_{0} \oplus g_{1}$. Then our remark follows from Lemma (11-1). Combining this with the fact

$$
\left\langle\left(R_{a \exp z}\right)_{*} X, \Theta_{-1}\right\rangle=\operatorname{Ad}\left(a^{-1}\right)\left\langle X, \Theta_{-1}\right\rangle
$$

our first assertion can be proved. From Lemma (11-4), differentiating (11-4), we have $0=\left[d \Theta_{0}, \Theta_{-1}\right]-\left[\Theta_{0}, d \Theta_{-1}\right]$. From (11-5), we have

$$
\begin{aligned}
{\left[\Theta_{-1}, \Omega_{0}\right] } & =\left[\Theta_{-1}, d \Theta_{0}\right]+\frac{1}{2}\left[\Theta_{-1},\left[\Theta_{0}, \Theta_{0}\right]\right]+\left[\Theta_{-1}\left[\Theta_{-1}, \Theta_{1}\right]\right] \\
& =\left[\Theta_{0}, d \Theta_{-1}\right]+\frac{1}{2}\left[\Theta_{-1}\left[\Theta_{0}, \Theta_{0}\right]\right]+\left[\Theta_{-1}\left[\Theta_{-1}, \Theta_{1}\right]\right] .
\end{aligned}
$$

Therefore the restriction of $\left[\Theta_{-1}, \Omega_{0}\right]$ onto $H_{q}$ is zero, this is nothing but $\partial c_{q}=0$. Analogously we can prove (3).

Let $\omega^{\prime}=\Theta_{-1} \oplus \Theta_{0}+\omega_{1}^{\prime}$ be another admissible Cartan connection on $Q(\mathfrak{a})$. As before we define $H_{q}^{\prime}$ by

$$
H_{q}^{\prime}=\left\{X \in T(Q)_{q} \mid\left\langle X, \Theta_{0}\right\rangle=0,\left\langle X, \omega_{1}^{\prime}\right\rangle=0\right\} .
$$

Take $u \in g_{-1}$. If $X_{u}$ (resp. $X_{u}^{\prime}$ ) is the element in $H_{q}$ (resp. $H_{q}^{\prime}$ ) such that $\left\langle X_{u}, \Theta_{-1}\right\rangle$ $=u$ (resp. $\left.\left\langle X_{u}^{\prime}, \Theta_{-1}\right\rangle=u\right)$, we have $\left\langle X_{u}-X_{u^{\prime}}^{\prime}, \Theta_{-1}\right\rangle=0$ and $\left\langle X_{u}-X_{u}^{\prime}, \Theta_{0}\right\rangle=0$. Therefore there exists an element $f_{q}(u)$ in $g_{1}$ such that $X_{u}-X_{u}^{\prime}=\left(f_{q}(u)\right)^{*}$. Clearly the mapping $f_{q}: \mathfrak{g}_{-1} \rightarrow \mathfrak{g}_{1}$ can be considered as an element of $C^{2,1}(\mathfrak{I})$. Thus with two admissible Cartan connections $\omega$ and $\omega^{\prime}$, we associate a function $f\left(\omega, \omega^{\prime}\right)$ from $Q$ into $C^{2,1}(\mathfrak{l})$.

Lemma (11-7). Let $\omega=\Theta_{-1} \oplus \Theta_{0} \oplus \omega_{1}$ and $\omega^{\prime}=\Theta_{-1} \oplus \Theta_{0} \oplus \omega_{1}^{\prime}$ be two admissible Cartan connections on $Q(\mathfrak{a})$. Then we have $c(\omega)_{q}-\left(c \omega^{\prime}\right)_{q}=\partial f\left(\omega, \omega^{\prime}\right)_{q}$ for each $q \in Q(\mathfrak{a})$.

Proof. Let $\Omega_{0}$ and $\Omega_{0}^{\prime}$ be the $g_{0}$-component of the curvature forms of $\omega$ and $\omega^{\prime}$ respectively. From (11-5) we have $\Omega_{0}-\Omega_{0}^{\prime}=\left[\Theta_{-1}, \omega_{1}-\omega_{1}^{\prime}\right]$. Take $u, v \in \mathfrak{g}_{-1}$. And 
let $X_{u}, Y_{v} \in H_{q}$ and $X_{u}^{\prime}, Y_{u}^{\prime} \in H_{q}^{\prime}$ be such that $\left\langle X_{n}, \Theta_{-1}\right\rangle=\left\langle X_{u}^{\prime}, \Theta_{-1}\right\rangle=u$ and $\left\langle Y_{v}, \Theta_{-1}\right\rangle=\left\langle Y_{v}^{\prime}, \Theta_{-1}\right\rangle=v$. Then

$$
\begin{aligned}
c(\omega)_{q}(u, v)-c\left(\omega^{\prime}\right)_{q}= & \left\langle X_{u} \wedge Y_{v}, \Omega_{0}\right\rangle-\left\langle X_{u}^{\prime} \wedge Y_{u}^{\prime}, \Omega_{0}^{\prime}\right\rangle \\
= & \left\langle X_{u}^{\prime}+\left(f\left(\omega, \omega^{\prime}\right)_{q}(u)\right)^{*} \wedge Y_{v}^{\prime}+\left(f\left(\omega, \omega^{\prime}\right)_{q}(v)\right)^{*}, \Omega_{0}\right\rangle \\
& -\left\langle X_{u}^{\prime} \wedge Y_{v}^{\prime}, \Omega_{0}^{\prime}\right\rangle \\
= & \left\langle X_{u}^{\prime} \wedge Y_{v}^{\prime}, \Omega_{0}\right\rangle-\left\langle X_{u}^{\prime} \wedge Y_{v}^{\prime}, \Omega_{0}^{\prime}\right\rangle \quad \text { (from Lemma (11-1)) } \\
= & \left\langle X_{u}^{\prime} \wedge Y_{v}^{\prime}, \Omega_{0}-\Omega_{0}^{\prime}\right\rangle=\left\langle X_{u}^{\prime} \wedge Y_{v}^{\prime},\left[\Theta_{-1}, \omega_{1}-\omega_{1}^{\prime}\right]\right\rangle .
\end{aligned}
$$

From this our assertion follows if we remark that

$$
\left\langle X_{u}^{\prime}, \omega_{1}-\omega_{1}^{\prime}\right\rangle=f\left(\omega, \omega^{\prime}\right)_{q}(u) \text { and }\left\langle Y_{v}^{\prime}, \omega_{1}-\omega_{1}^{\prime}\right\rangle=f\left(\omega, \omega^{\prime}\right)_{q}(v) .
$$

The function $c(\omega)$ from $Q(\mathfrak{a})$ into $C^{\mathbf{1 , 2}}(\mathfrak{l})$ is called Weyl cocycle for $\omega$.

TheOREM (11-1) (N. TANAKA). Let $L / L_{0}$ be a semisimple flat homogeneous space such that $H^{2,1}(\mathfrak{l})=0$. Given a $G_{0}$-structure $P$ on a manifold $M$, here $G_{0}$ denotes the linear isotropy group of $L / L_{0}$. Then for each $L_{0}$-equivalence class $a$ in the set of all $G_{0}$-connections on $P$, there exists uniquely an admissible Cartan connection on the associated $L_{0}$-structure $Q(a)$ of a such that its Weyl cocycle $c(\omega)_{q}$ is harmonic at each point $q$ of $Q(\mathfrak{a})$ (cf., the definition after Lemma (4-1)).

Proof. (Uniqueness). Let $\omega=\Theta_{-1} \oplus \Theta_{0} \oplus \omega_{1}$ and $\omega^{\prime}=\Theta_{-1}+\Theta_{0} \oplus \omega_{1}^{\prime}$ be two admissible Cartan connections such that their Weyl cocycle $c(\omega)$ and $c\left(\omega^{\prime}\right)$ are harmonic, or equivalently $\partial c(\omega)=\partial^{*} c\left(\omega^{\prime}\right)=0$. From Lemma (11-7), we have $c(\omega)-c\left(\omega^{\prime}\right)=\partial f\left(\omega, \omega^{\prime}\right)$. Thus we have $\partial^{*} \partial f\left(\omega, \omega^{\prime}\right)=\square f\left(\omega, \omega^{\prime}\right)=0$. Since $H^{2,1}(\mathfrak{l})$ $=0$, we have $f\left(\omega, \omega^{\prime}\right)=0$. This is nothing but $\omega_{1}=\omega_{1}^{\prime}$.

(Existence). Let $\bar{\omega}=\Theta_{-1} \oplus \Theta_{0} \oplus \bar{\omega}_{1}$ be an arbitrary admissible Cartan connection on $Q(\mathfrak{a})$ (cf. Lemma (11-3)). Let $J$ be a $C^{2,1}(\mathfrak{l})$-valued function on $Q(\mathfrak{a})$ determined by $\square J=\partial^{*} \partial J=\partial^{*} c(\omega) . J$ is well determined since $H^{2,1}(\mathfrak{l})=0$ implies that $\square$ is an isomorphism of $C^{2,1}(\mathfrak{l})$. For $a \cdot \exp z \in L_{0}\left(a \in G_{0}, z \in \mathfrak{g}_{1}\right)$, we have $J_{q \cdot a \exp z}=\rho(a)^{-1} J_{q}$ since $\rho(a) \square=\square \rho(a)$ and $c(\omega)_{q \cdot a \cdot \exp z}=\rho(a) c(\omega)_{q}$ (cf. Lemma (11-6)). We define a I-valued 1-form $\omega$ on $Q(\mathfrak{a})$ by

$$
\langle X, \omega\rangle=\langle X, \bar{\omega}\rangle+J\left(\left\langle X, \Theta_{-1}\right\rangle\right),
$$

for a vector tangent to $Q$. Then $\omega$ is the required admissible Cartan connection. In fact

(i) $\left\langle A^{*}, \omega\right\rangle=\left\langle A^{*}, \omega\right\rangle+J\left(\left\langle A^{*}, \Theta_{-1}\right\rangle\right)=\left\langle A^{*}, \omega\right\rangle=A$.

(ii) If $\langle X, \omega\rangle=0$, then

$$
0=\langle X, \omega\rangle+J\left(\left\langle X, \Theta_{-1}\right\rangle\right)=\left\langle X, \Theta_{-1}\right\rangle+\left\langle X, \Theta_{0}\right\rangle+\left\{\left\langle X, \bar{\omega}_{1}\right\rangle+J\left(\left\langle X, \Theta_{-1}\right\rangle\right)\right\} .
$$

Thus $\left\langle X, \Theta_{-1}\right\rangle=\left\langle X, \Theta_{0}\right\rangle=0$. Therefore $X$ is of the form $A^{*}$ for some $A \in \mathfrak{g}_{1}$. Since $\left\langle A^{*}, \omega\right\rangle=A, A$ must be 0 . 
(iii) For $a \cdot \exp z \in L_{0}\left(a \in G_{0}, z \in \mathfrak{g}_{1}\right)$ and a vector $X_{q}$ in $T(Q)_{q}$, we have

$$
\begin{aligned}
\left\langle X_{q},\left(R_{a \cdot \exp z}\right)^{*} \omega\right\rangle & =\left\langle\left(R_{a \cdot \exp z}\right)_{*} X, \omega\right\rangle \\
& =\left\langle\left(R_{a \cdot \exp z}\right)_{*} X_{q}, \omega\right\rangle+J_{q \cdot a \cdot \exp z}\left(\left\langle\left(R_{a \cdot \exp z}\right)_{*}, \Theta_{-1}\right\rangle\right) \\
& =\operatorname{Ad}(a \exp z)^{-1}\left\langle X_{q}, \omega\right\rangle+J_{q \cdot a \cdot \exp z}\left(\operatorname{Ad}\left(a^{-1}\left\langle X, \Theta_{-1}\right\rangle\right)\right) \\
& =\operatorname{Ad}(a \exp z)^{-1}\left\langle X_{q}, \omega\right\rangle+\operatorname{Ad}\left(a^{-1}\right)\left(\rho(a) J_{q, a \exp z}\right)\left(\left\langle X, \Theta_{-1}\right\rangle\right) \\
& =\operatorname{Ad}(a \exp z)^{-1}\left\langle X_{q}, \omega\right\rangle+\operatorname{Ad}\left(a^{-1}\right) \cdot J_{q}\left(\left\langle X, \Theta_{-1}\right\rangle\right) \\
& =\operatorname{Ad}(a \exp z)^{-1}\left\langle X_{q}, \omega\right\rangle+\operatorname{Ad}(a \exp z)^{-1} \cdot J_{q}\left(\left\langle X, \Theta_{-1}\right\rangle\right) \\
& =\operatorname{Ad}(a \exp z)^{-1}\left\langle X_{q}, \omega\right\rangle .
\end{aligned}
$$

Thus we have shown that $\omega$ is a Cartan connection. Clearly this is admissible. Now the Weyl cocycle $c(\omega)$ of $\omega$ is given by $c(\omega)=c(\omega)-\partial J$. Therefore $\partial^{*} c(\omega)$ $=\partial^{*} c(\omega)-\partial^{*} \partial J$. Since $\partial^{*} \partial J=\partial^{*} c(\omega)$, we have $\partial^{*} c(\omega)=0$, i.e. $c(\omega)=0$.

The admissible Cartan connection, which is assured to exist in Theorem (11-1) is called the normal Cartan connection of the $L_{0}$-equivalence class.

Now we preserve our notations in Theorem (11-1). Let $H: M \rightarrow M$ be a $G_{0^{-}}$ automorphism of $P$, preserving $\mathfrak{a}$. Then 2 nd prolongation $h^{(2)}$ of $h$ is an isomorphism of $Q(\mathfrak{a})$. Then by the uniqueness, $h^{(2)}$ is an isomorphism of the normal Cartan connection. Thus we have a natural mapping from the group of $G_{0}$-automorphism preserving $\mathfrak{a}$ into the group of isomorphisms of the normal Cartan connections.

LEMMA (11-8). The mapping defined above is bijective.

Proof. The injectivity is trivial. The surjectivity follows from Lemma (8-4).

COROLlaRY (11-1). The group of all $G_{0}$-automorphisms of $P$ preserving $a$ is a finite dimensional Lie group.

Proof. This follows from Lemma (11-8) and the fact that the automorphism group of a Cartan connection is a finite dimensional Lie group [11].

Now let $L \rightarrow L / L_{0}$ be the flat $L_{0}$-structure. And as in $\S 9, \Xi=\Xi_{-1} \oplus \Xi_{0} \oplus \Xi_{1}$ be the Mauer-Cartan form of $L$. Then $\Xi$ is clearly the normal Cartan connection of the natural equivalence class on the flat $G_{0}$-structure.

LEMMA (11-9). The automorphism group of the normal Cartan connection of $L \rightarrow L / L_{0}$ is $L$.

Proof. Clearly $L$ is contained in the automorphism group since $L$ preserves the Mauer-Cartan form. Conversely let $h$ be a bundle isomorphism preserving the normal Cartan connection. Then $h^{(2)}$ preserves the Mauer-Cartan connection. Then $L_{h}^{(2)}(e)^{-1} \cdot h^{(2)}$ also preserves the Mauer-Cartan form and the identity of $L$. Therefore $L_{h}^{(2)}(e)^{-1} \cdot h$ is the identity on the connected component of the identity. Then $h^{(2)}$ is $L_{h^{(2)}}{ }_{(e)}$ from Lemma (8-4).

LeMmA (11-10). A local automorphism of the normal Cartan connection of $L \rightarrow L / L_{0}$ can be uniquely extended to a global automorphism of $L \rightarrow L / L_{0}$. 
Proof. This can be proved as Lemma (11-9).

12. The Weyl tensors. Let $L / L_{0}$ be a semisimple flat homogeneous space such that $H^{2,1}(\mathfrak{l})=0$. Given a $G_{0}$-structure $P$ on a manifold $M$, here $G_{0}$ is the linear isotropy subgroup of $L / L_{0}$. Let $\dot{\mathfrak{a}}$ be a $L_{0}$-equivalence class in $A(P)$, the set of all $G_{0}$-connection on $P$ without torsion. Let $\omega$ be the normal Cartan connection on $Q(\mathfrak{a})$; the associated $L_{0}$-structure of $\mathfrak{a}$. Then by Lemma (11-1) and Lemma (11-6), the Weyl cocycle $c(\omega)$ can be considered as a tensor field of type (1.3). We call the tensor field the Weyl tensor field of the family a, and denoted by $W(\mathfrak{a})$. If $L / L_{0}$ satisfies furthermore $H^{1,1}(\mathfrak{l})=0$, we can uniquely define an $L_{0}$-structure of 2 nd order since there is only one equivalence class in $A(P)$ (see Proposition (10-1)). In this case we call $W(\mathfrak{a})$ the Weyl tensor of a $G_{0}$-structure $P$.

THEOREM (12-1). Let $L / L_{0}$ be a semisimple flat homogeneous space such that $H^{2,1}(\mathfrak{l})=0$ and $H^{2,2}(\mathfrak{l})=0$. Given an $L_{0}$-equivalence class a on a $G_{0}$-structure $P$, $G_{0}$ being the linear isotropy subgroup of $L / L_{0}$. Then the following are equivalent to each other:

(1) $W(\mathfrak{a})=0$,

(2) $Q(\mathfrak{a})$ is flat.

If $L / L_{0}$ satisfies $H^{1,1}(\mathfrak{l})=0$, then the following are equivalent to each other:

(1') The Weyl tensor of $P$ vanishes.

(2') $P$ is flat.

Proof. Since the Weyl tensor of the flat $L_{0}$-structure $L \rightarrow L / L_{0}$ vanishes, (2) clearly implies (1). By Lemma (11-6), $W(\mathfrak{a})=0$ implies the curvature form of the normal Cartan connection vanishes since $H^{2,2}(\mathfrak{l})=0$. Then by Proposition (9-1), (1) implies (2). The second assertion follows from Lemma (7-4).

Proposition (12-1). Let $L / L_{0}$ be a semisimple flat homogeneous space such that $H^{2,1}(\mathfrak{l})=0$. Let $\Gamma$ be a $G_{0}$-connection in a $L_{0}$-equivalence class $\mathfrak{a}$ in $A(P)$. We denote by $s$ the admissible cross-section corresponding to $\Gamma$. Let $\omega=\Theta_{-1} \oplus \Theta_{0} \oplus \omega_{1}$ be the normal Cartan connection of $a$.

(1) There exists a tensor field $S: P \rightarrow C^{2,1}(\mathfrak{l})$ such that $\left\langle X, s^{*} \omega_{1}\right\rangle=S(\langle X, \theta\rangle)$ for a vector tangent to $P$, here $\theta$ is the canonical form of $P$.

(2) $W(\mathfrak{a})=\mathfrak{r}(\Gamma) \oplus \partial S, \mathfrak{r}(\Gamma)$ is the curvature tensor field of $\Gamma$.

(3) $S=-\square^{-1}\left(\partial^{*}(\Gamma)\right)$.

Proof. Since $s^{*} \omega$, is zero on the fibre of $P \rightarrow M, s^{*} \omega$ can be expressed by $\theta$. (2) From (11-5), we have

$$
\begin{aligned}
s^{*} \Omega_{0} & =s^{*}\left(d \Theta_{0}+\frac{1}{2}\left[\Theta_{0}, \Theta_{0}\right]+\left[\Theta_{-1}, \omega_{1}\right]\right) \\
& =d s^{*} \Theta_{0}+\frac{1}{2}\left[s^{*} \Theta_{0}, s^{*} \Theta_{0}\right]+\left[s^{*} \Theta_{-1}, s^{*} \omega_{1}\right] .
\end{aligned}
$$

Since $s^{*} \omega_{0}=\Gamma, s^{*} \Theta_{-1}=\theta$, we get $s^{*} \Omega_{0}=\mathfrak{r}(\Gamma)+\left[\theta, s^{*} \omega_{1}\right]$. From this (2) is proved. (3) Applying $\partial^{*}$ to (2), we have $\partial^{*} W(\mathfrak{a})=\partial^{*} \mathfrak{r}(\Gamma)+\partial^{*} \partial S$. Since $\partial^{*} W(\mathfrak{a})=0$, we have the desired formula. 
13. Applications to geometric transformations. Let $L / L_{0}$ be a semisimple flat homogeneous space such that $H^{2,1}(\mathfrak{l})=0$. Given a $G_{0}$-structure $P$ on a manifold $M, G_{0}$ being the linear isotropy subgroup of $L / L_{0}$. Let $\mathfrak{a}$ be an $L_{0}$-equivalence class in $A(P)$. Let $\Omega=\Omega_{0}+\Omega_{1}$ be the curvature form of the normal Cartan connection of a. Let $s: P \rightarrow Q(\mathfrak{a})$ be an admissible cross-section, and $\Gamma$ be the $G_{0}$-connection corresponding to $s$. Then $\partial^{*}(\mathfrak{r}(\Gamma))$ is called the Ricci tensor field of $\Gamma$, here $\mathfrak{r}(\Gamma)$ is the curvature tensor field of $\Gamma$. Here we remark that $g_{1}$ and $g_{-1}$ are naturally dual from Lemma (3-3), therefore we can consider $\partial^{*} \mathfrak{r}(\Gamma)$ as a tensor field.

THEOREM (13-1). Let $s: P \rightarrow Q(\mathfrak{a})$ be a $G_{0}$-connection in a. If the Ricci tensor field is parallel $\left(\right.$ i.e. $\left.\nabla\left(\partial^{*} \mathfrak{r}(\Gamma)\right)=0\right)$, then $s^{*} \Omega_{1}=0$.

Proof. From (11-6), we have

$$
s^{*} \Omega_{1}=s^{*}\left(d \omega_{1}+\left[\omega_{1}, \Theta_{0}\right]\right)=d s^{*} \omega_{1}+\left[s^{*} \omega_{1}, \Gamma\right] \quad\left(\Gamma=s^{*} \Theta_{0}\right) .
$$

Since $s^{*} \Omega_{1}$ is zero on the fibres of $P \rightarrow M$, for tangent vectors $X$ and $Y$, we have

$$
\begin{aligned}
\left\langle X \wedge Y, s^{*} \Omega_{1}\right\rangle & =\left\langle V_{\theta(X)} \wedge V_{\theta(Y)}, s^{*} \Omega_{1}\right\rangle=\left\langle V_{\theta(X)} \wedge V_{\theta(Y)}, d s^{*} \omega_{1}\right\rangle \\
& =V_{\theta(X)}\left\langle V_{\theta(Y)}, s^{*} \omega_{1}\right\rangle-V_{\theta(Y)}\left\langle V_{\theta(X)}, s^{*} \omega_{1}\right\rangle-\left\langle\left[V_{\theta(X)}, V_{\theta(Y)}\right], s^{*} \omega_{1}\right\rangle .
\end{aligned}
$$

Here $V_{\theta(X)}$ and $V_{\theta(Y)}$ denote the horizontal vector fields corresponding $\theta(X)$ and $\theta(Y)$ respectively. Since $\Gamma$ is torsion free, $\left[V_{\theta(X)}, V_{\theta(Y)}\right]$ is tangent to the fibres. Thus we get

$$
\left\langle V_{u} \wedge V_{v}, s^{*} \Omega_{1}\right\rangle=\left(\nabla_{u} S\right)(v)-\left(\nabla_{v} S\right)(u)
$$

for $u, v \in \mathfrak{g}_{-1}$, here $S$ is defined in Proposition (12-1). By Proposition (12-1), we know $S=-\square^{-1}\left(\partial^{*} \mathfrak{r}(\Gamma)\right)$. In particular $\nabla \partial^{*} \mathfrak{r}(\Gamma)=0$ implies $\nabla S=0$. Thus $s^{*} \Omega_{1}=0$.

TheOREM (13-2). Let $s: P \rightarrow Q(\mathfrak{a})$ and $t: P \rightarrow Q(\mathfrak{a})$ be two $G_{0}$-connections in a. Then there exists a tensor field $A: P \rightarrow g_{1}$ such that $A(p)=s(p) \cdot \exp A_{p}$. If the Ricci tensor fields of $s$ and $t$ are parallel, then $\left[A_{p}, W(\mathfrak{a})_{p}\right]=0$ for $p \in P$.

Proof. The first assertion is easy to see. For tangent vectors $X_{p}$ and $Y_{p}$ at $p$, we have

$$
\begin{aligned}
\left\langle X_{p} \wedge Y_{p}, t^{*} \Omega_{\mathrm{i}}\right\rangle & =\left\langle X_{p} \wedge Y_{p},\left(R_{\exp A \cdot \mathrm{s}}\right)^{*} \Omega_{1}\right\rangle \\
& =\left\langle\left(R_{\exp A \cdot s}\right)_{*}\left(X_{p} \wedge Y_{p}\right), \Omega_{1}\right\rangle .
\end{aligned}
$$

Since $\left(R_{\exp A \cdot s}\right)_{*} X_{p}=\left(R_{\exp A}\right)_{*}\left(s_{*} X_{p}\right)+\{$ a vertical vector $\}$, we have

$$
\left\langle X_{p} \wedge Y_{p}, t^{*} \Omega_{1}\right\rangle=\left\langle\left(R_{\exp A_{p}}\right)_{*} S_{*}\left(X_{p} \wedge Y_{p}\right), \Omega_{1}\right\rangle \text { from Lemma (11-1). }
$$

Then by Lemma (11-2), we have

$$
\begin{aligned}
\left\langle X_{p} \wedge Y_{p}, t^{*} \Omega_{1}\right\rangle & =\left\langle s_{*}\left(X_{p} \wedge Y_{p}\right),-\left[A_{p}, \Omega_{0}\right]+\Omega_{1}\right\rangle \\
& =\left\langle X_{p} \wedge Y_{p},-\left[A_{p}, s^{*} \Omega_{0}\right]+s^{*} \Omega_{1}\right\rangle .
\end{aligned}
$$

Since $s^{*} \Omega_{1}$ and $t^{*} \Omega_{1}=0$, we have $\left[A_{p}, s^{*} \Omega_{0}\right]=0$. Since $s^{*} \Omega_{0}$ defines the Weyl tensor $W(\mathfrak{a})$, we have the desired result. 
For a $G_{0}$-connection $\Gamma$ in $\mathfrak{a}$, we denote by $\operatorname{Aut}(P, \Gamma)$ the subgroup of the automorphism group Aut (a) of the $L_{0}$-equivalence class a preserving $\Gamma$. We suspect:

Conjecture. We assume $L / L_{0}$ is simple. If $\Gamma$ is complete, Ricci-parallel and Aut $(P, \Gamma)$ is strictly different from Aut (a), then (up to covering) $M$ is diffeomorphic to $L / L_{0}$ and $L_{0}$-structure $Q(\mathfrak{a}) \rightarrow M$ coincides with the flat $L_{0}$-structure $L \rightarrow L / L_{0}$. This conjecture is true when $L / L_{0}$ is a projective space (resp. a Moebius space) and $L$ is the group of projective transformations (resp. conformal transformations), provided $\Gamma$ is the Lemi-Civita connection of some riemannian metric [20], [21].

THEOREM (13-3). Let $L / L_{0}$ be a simple flat homogeneous space such that $H^{2,1}(\mathfrak{l})=0$. Given a $G_{0}$-structure $P$ on $M, G_{0}$ being the linear isotropy group of $L / L_{0}$, let $\Gamma$ be a complete $G_{0}$-connection on $P$ whose Ricci-tensor is parallel. If we have

(1) Aut $(\{\Gamma\})$ is transitive on $M$,

(2) the connected component of the linear isotropy group is irreducible,

(3) Aut $(P,\{\Gamma\})=$ Aut $(\{\Gamma\})$,

then $M$ is diffeomorphic to $L / L_{0}$ (up to covering) and Aut $(P,\{\Gamma\})$ is identical to $L$.

Proof. We may assume $M$ is simply connected. Let $f$ be an element in Aut $(\{\Gamma\})$ such that $f^{*} \Gamma \neq \Gamma$. Let $p \in P$ be a point such that $f^{*} \Gamma_{p} \neq \Gamma_{p}$. Taking as $s$ and $t$ in Theorem (13-2) the cross-sections corresponding to $f^{*} \Gamma$ and $\Gamma$ respectively, we get the tensor field $A$ such that $\left[A_{p}, W\left(\{\Gamma)_{p}\right]=0\right.$. We denote by $\operatorname{Ad}(g)$ the linear isotropy representation of the isotropy subgroup Aut $(\{\Gamma\})_{p}$ of Aut $(\{\Gamma\})$ at $p$. For each $g$ in Aut $(\{\Gamma\})_{p}$, replacing $f$ by $g$ we also have

$$
\left[{ }^{t} \operatorname{Ad}(g)^{-1} A_{p}, W(\{\Gamma\})_{p}\right]=0 .
$$

Since the linear isotropy representation is irreducible, we have $W(\{\Gamma\})_{p}=0$. Then the homogeneity implies $W(\{\Gamma\})=0$. By Proposition (12-1), we have

$$
\mathfrak{r}(\Gamma)=\partial\left(\square^{-1}(\partial * \mathfrak{r}(\Gamma))\right)
$$

Since the Ricci-tensor is parallel, so is the curvature tensor $\mathfrak{r}(\Gamma)$. Thus $(M, \Gamma)$ is a global affine symmetric space, and so is $\left(M, f^{*} \Gamma\right)$. Let $x$ be a point in $M$ and $\tau_{x}$ be the symmetry of $(M, \Gamma)$ at $x$. If $G_{0}$ contains the element $-I$, then $\tau_{x}$ preserves $P$ since $\Gamma$ is a $G_{0}$-connection on $P$ and $\tau_{x}$ preserves the fibre of $P$ at $x$. When $G_{0}$ does not contain $-I$, we consider the bundle extension to $P \cup P \cdot(-I)$ (disjoint). Then $\tau_{x}(P)=P \cdot(-I)$ and $\tau_{x}(P \cdot(-I))=P$. Now there exists a point $z$ in $M$ such that $\tau_{z} \cdot \tau_{z} \neq$ id here $\tau_{z}^{\prime}$ is the symmetry of $\left(M, f^{*} \Gamma\right)$ at $z$. In fact, otherwise, the symmetries of $\left(M, f^{*} \Gamma\right)$ coincide at every point $M$. From this we see that $\Gamma=f^{*} \Gamma$. Then from the above remark, we see $\tau_{z} \tau_{z}^{\prime}$ preserves $P$. Then it is easy to see that $\tau_{z} \tau_{z}^{\prime}$ is an element of Aut $(\{\Gamma\})$. Let $\mathfrak{h}$ be the Lie algebra of Aut $(\{\Gamma\})$. Then $\mathfrak{h}$ can be considered as a germ of vector field at $z$. Since $Q(\{\Gamma\})$ is locally equivalent to $L / L_{0}$, we can consider $\mathfrak{h}$ is a germ of vector field at the origin of $L / L_{0}$. Then using Lemma 
(11-10), we can consider $\mathfrak{h}$ is a subalgebra of $\mathfrak{l}$. Also using (11-10), $\tau_{z} \cdot \tau_{z}^{\prime}$ can be considered as an element of $L$. Furthermore $\tau_{z} \cdot \tau_{1}^{\prime}$ belongs to exp $\mathfrak{g}_{1}$ since its linear isotropy representation is the identity. We write $\tau_{z} \tau_{z}^{\prime}$ as $\tau_{z} \cdot \tau_{z}^{\prime}=\exp Z(Z \neq 0)$. Then clearly $\mathfrak{h} \oplus\{Z\}$ forms a subalgebra of $\mathfrak{l}$ such that its linear isotropy representation is irreducible. Then using Proposition (3-6), we conclude, $\mathfrak{h}=\mathfrak{l}$. Therefore $M=L / L_{0}$.

\section{BIBLIOGRAPHY}

1. A. Borel, On the curvature tensor of the Hermitian symmetric manifolds, Ann. of Math. (2) 71 (1960), 508-521. MR 22 \#1923.

2. A. Borel and F. Hirzebruch, Characteristic classes and homogeneous spaces. I, Amer. J. Math. 80 (1958), 458-538. MR 21 \#1586.

3. E. Cartan, "Les espaces a connexion conforme," en Oevres complètes. Part III, Vol. I: Divérs, géométrie différentielle, Gauthier-Villars, Paris, 1955, pp. 749-797. MR 17, 697.

4. - "Sur les variétés a connexion projective," en Oevres complètes. Part III, Vol. I: Divérs, géométrie différentielle, Gauthier-Villars, Paris, 1955, pp. 825-861. MR 17, 697.

5. S. S. Chern, Pseudo-groupes continus infinis, géométrie différentielle, Colloq. Internat. Centre National Recherche Scientifique (Strasbourg, 1953) Centre National de la Recherche Scientifique, Paris, 1953, pp. 119-136. MR 16, 112.

6. C. Ehresmann, Les connexions infinitésimales dans un espace fibré différentiable, Colloque de Topologie (espaces fibrés) (Bruxelles, 1950) Georges Thone, Liège et Masson, Paris, 1951, pp. 29-55. MR 13, 159.

7. V. W. Guillemin, The integrability problem for G-structures, Trans. Amer. Math. Soc. 116 (1965), 544-560. MR 34 \#3475.

8. V. W. Guillemin and S. Sternberg, Deformation theory of pseudogroup structures, Mem. Amer. Math. Soc. No. 64 (1966). MR 35 \#2302.

9. S. Helgason, Differential geometry and symmetric spaces, Pure and Appl. Math., vol. 12, Academic Press, New York, 1962. MR 26 \#2986.

10. S. Kobayashi, On connections of Cartan, Canad. J. Math. 8 (1956), 145-156. MR 17, 1126.

11. - Theory of connections, Ann. Math. Pura Appl. (4) 43 (1957), 119-194. MR 20 \#2760.

12. - Canonical forms on frame bundles of higher order contact, Proc. Sympos. Pure Math., vol. 3, Amer. Math. Soc., Providence, R. I., 1961, pp. 186-193. MR 23 \#A4104.

13. S. Kobayashi and T. Nagano, On projective connections, J. Math. Mech. 13 (1964), 215-235. MR 28 \#2501.

14. — On filtered Lie algebras and geometric structures. I, II, J. Math. Mech. 13 (1964), 875-907; ibid., 14 (1965), 513-521. MR 29 \#5961; MR 32 \#2512.

15. S. Kobayashi and K. Nomizu, Foundations of differential geometry. Vol. I, Interscience, New York, 1963. MR 27 \#2945.

16. B. Kostant, Lie algebra cohomology and the generalized Borel-Weil theorem, Ann. of Math. (2) 74 (1961), 329-387. MR 26 \#265.

17. Y. Matsushima and S. Murakami, On vector bundle valued harmonic forms and automorphic forms on symmetric riemannian manifolds, Ann. of Math. (2) 78 (1963), 365-416. MR 27 \#2997.

18. - On certain cohomology groups attached to Hermitian symmetric spaces, Osaka J. Math. 2 (1965), 1-35. MR 32 \#1728.

19. S. Murakami, Cohomology groups of vector-valued forms on symmetric spaces, Lecture Note, Univ. of Chicago, Chicago, Ill., 1966.

20. T. Nagano, The projective transformation on a space with parallel Ricci tensor, Kōdai Math. Sem. Rep. 11 (1959), 131-138. MR 22 \#216. 
21. T. Nagano, The conformal transformation on a space with parallel Ricci tensor, J. Math. Soc. Japan 11 (1959), 10-14. MR 23 \#A1330.

22. - Transformation groups on compact symmetric spaces, Trans. Amer. Math. Soc. 118 (1965), 428-353. MR 32 \#419.

23. T. Ochiai, Classification of the finite nonlinear primitive Lie algebras, Trans. Amer. Math. Soc. 124 (1966), 313-322. MR 34 \#4320.

24. —, On the automorphism group of a G-structure, J. Math. Soc. Japan 18 (1966), 189-193. MR 33 \#3224.

25. "Sophus Lie" de l'École Normale Supérieure 1954/55, Séminaire théorie des algèbres de Lie. Topologie des groupes de Lie, Secrétariat mathématique, Paris, 1955. MR 17, 384.

26. I. M. Singer and S. Sternberg, The infinite group of Lie and Cartan. I. The transitive groups, J. Analyse Math. 15 (1965), 1-114. MR 36 \#911.

27. N. Tanaka, Projective connections and projective transformations, Nagoya Math. J. 12 (1957), 1-24. MR 21 \#3899.

28. - Conformal connections and conformal transformations, Trans. Amer. Math. Soc. 92 (1959), 168-190. MR 23 \#A1331.

29. - On the equivalence problems associated with a certain class of homogeneous spaces, J. Math. Soc. Japan 17 (1965), 103-139. MR 32 \#6358.

30. H. Weyl, Zur Infinitesimalgeometrie Einordnung der projektiven und der konformen Auffassung, Göttingen. Nachr. 1921, 99-112.

UNIVERSITY OF NOTRE DAME,

Notre DAME, INDIANA 46556 